\title{
INTRODUCTION OF A COMPLEX STRUCTURE ON THE $p$-INTEGRABLE TEICHMÜLLER SPACE
}

\author{
Masahiro Yanagishita \\ Waseda University, Departments in Fundamental Science and Engineering \\ 3-4-1 Okubo, Shinjuku, Tokyo 169-8555, Japan; m-yanagishita@asagi.waseda.jp
}

\begin{abstract}
For $p \geq 1$, the $p$-integrable Teichmüller space is the metric subspace of the Teichmüller space composed of the Teichmüller equivalence classes with $p$-integrable Beltrami coefficient. In this paper, for $p \geq 2$, we introduce a complex structure on the $p$-integrable Teichmüller space of an arbitrary Fuchsian group satisfying a certain geometric condition. As an application, we show the coincidence of two canonical distances on the metric subspace.
\end{abstract}

\section{Introduction}

Let $\Gamma$ be a Fuchsian group acting on the unit disk $\Delta=\{|z|<1\}$ of the complex plane $\mathbf{C}$ and $\operatorname{Bel}(\Gamma)$ be the open unit ball of the Banach space of measurable $(-1,1)$ differentials on $\Delta$ for $\Gamma$ with finite $L^{\infty}$-norm

$$
\|\mu\|_{\infty}=\underset{z \in \Delta}{\operatorname{ess} \sup }|\mu(z)| \text {. }
$$

Here a function $\mu$ on $\Delta$ is a $(-1,1)$-differential for $\Gamma$ if

$$
(\mu \circ \gamma) \frac{\overline{\gamma^{\prime}}}{\gamma^{\prime}}=\mu
$$

for every $\gamma \in \Gamma$. Each element of $\operatorname{Bel}(\Gamma)$ is called a Beltrami coefficient for $\Gamma$. For $\mu, \nu \in \operatorname{Bel}(\Gamma), \mu$ is Teichmüller equivalent to $\nu$ if

$$
\left.f^{\mu}\right|_{\partial \Delta}=\left.f^{\nu}\right|_{\partial \Delta}
$$

where $f^{\mu}$ is the quasiconformal self-mapping on $\Delta$ with Beltrami coefficient $\mu$ and fixes $1, i$ and -1 . The Teichmüller space $T(\Gamma)$ of $\Gamma$ is the quotient space of $\operatorname{Bel}(\Gamma)$ by the Teichmüller equivalence relation. Let $\varpi$ be the quotient map of $\operatorname{Bel}(\Gamma)$ onto $T(\Gamma)$ and $[\mu]$ be the Teichmüller equivalence class represented by $\mu \in \operatorname{Bel}(\Gamma)$. Especially, the Teichmüller equivalence class represented by the zero function on $\Delta$ is called the base point of $T(\Gamma)$, denoted by 0 .

For $p \geq 1$, a Beltrami coefficient $\mu \in \operatorname{Bel}(\Gamma)$ is $p$-integrable if $\mu$ has a finite hyperbolic $L^{p}$-norm

$$
\|\mu\|_{p}=\left(\iint_{N}|\mu(z)|^{p} \rho(z)^{2} d x d y\right)^{\frac{1}{p}},
$$

where $N$ is a fundamental region on $\Delta$ for $\Gamma$ and $\rho(z)=\left(1-|z|^{2}\right)^{-1}$ is the Poincaré metric on $\Delta$. Let $\operatorname{Ael}^{p}(\Gamma)$ be the set of $p$-integrable Beltrami coefficients. A Teichmüller equivalence class $\tau \in T(\Gamma)$ is $p$-integrable if $\tau$ has a $p$-integrable Beltrami coefficient

doi:10.5186/aasfm.2014.3952

2010 Mathematics Subject Classification: Primary 30F60; Secondary 32G15, 30C60.

Key words: Teichmüller spaces, quasiconformal mappings, Douady-Earle extension. 
as its representative. The $p$-integrable Teichmüller space $T^{p}(\Gamma)$ of $\Gamma$ is defined as the subset of $T(\Gamma)$ composed of $p$-integrable Teichmüller equivalence classes.

Let $\Delta^{*}$ be the exterior of $\Delta$ in the extended complex plane $\hat{\mathbf{C}}$. It is known that for every Fuchsian group $\Gamma, T(\Gamma)$ has a complex structure modeled on the Banach space $\mathcal{B}(\Gamma)$ of holomorphic quadratic differentials on $\Delta^{*}$ for $\Gamma$ with finite hyperbolic $L^{\infty}$-norm

$$
\|\varphi\|_{\infty}=\sup _{z \in \Delta^{*}}|\varphi(z)| \rho_{*}(z)^{-2}
$$

where $\rho_{*}(z)=\left(|z|^{2}-1\right)^{-1}$ is the Poincaré metric on $\Delta^{*}$. This fact results from a homeomorphism of $T(\Gamma)$ into $\mathcal{B}(\Gamma)$ called the Bers embedding. In this paper, we prove that the $p$-integrable Teichmüller space $T^{p}(\Gamma)$ has a complex structure if $p \geq 2$ and $\Gamma$ has a certain geometric condition.

Let $A^{p}(\Gamma)$ be the Banach space of holomorphic quadratic differentials on $\Delta^{*}$ for $\Gamma$ with finite hyperbolic $L^{p}$-norm

$$
\|\varphi\|_{p}=\left(\iint_{N^{*}}|\varphi(z)|^{p} \rho_{*}(z)^{2-2 p} d x d y\right)^{\frac{1}{p}} .
$$

Here $N^{*}$ is a fundamental region on $\Delta^{*}$ for $\Gamma$. What we have to show is that the Bers embedding is a homeomorphism of $T^{p}(\Gamma)$ into $A^{p}(\Gamma)$. We prepare for the proof in Section 2. Specifically, we characterize each point of $T^{p}(\Gamma)$ by its Douady-Earle extension, which is a quasiconformal self-mapping on $\Delta$ with conformal naturality (see [6]). Originally, Cui [5] proved this result in the case of $T^{2}(1)$ and Tang [20] extended it to $T^{p}(1)$ for $p \geq 2$, where $1=\left\{\operatorname{id}_{\Delta}\right\}$ is the trivial group. In the proof, they applied the Dirichlet integral of harmonic self-maps on $\Delta$ obtained by the Poisson integral (see [4]). We extend their arguments to the case of every Fuchsian group by combining the invariant formula of the Bergmann kernel and a partition of the integral in $\Delta$, that is,

$$
\iint_{\Delta} \cdots d x d y=\sum_{\gamma \in \Gamma} \iint_{\gamma(N)} \cdots d x d y .
$$

In Section 3, we prove the continuity of the Bers embedding $\beta: T^{p}(\Gamma) \rightarrow A^{p}(\Gamma)$. The proof is composed of two steps. One is to compare between the distance on $\mathrm{Ael}^{p}(\Gamma)$ induced by norm (1.2) and the one on $\beta\left(T^{p}(\Gamma)\right)$ induced by norm (1.3). The other is to show the continuity of the self-map of $\operatorname{Ael}^{p}(\Gamma)$ that maps each $\mu \in \operatorname{Ael}^{p}(\Gamma)$ to the Beltrami coefficient of the Douady-Earle extension with boundary value $\left.f^{\mu}\right|_{\partial \Delta}$. In Section 4 , we show the continuity of the inverse map $\beta^{-1}$ by the similar way to the whole space $T(\Gamma)$. Indeed, we construct a local continuous section for each point in $\beta\left(T^{p}(\Gamma)\right)$. For the proof, we need to assume the geometric condition for $\Gamma$ we mentioned previously.

In Section 5, we introduce the right translation map for each point $\tau$ in the Teichmüller space, which is a homeomorphism between two Teichmüller spaces and switching $\tau$ to the base point (see [16], Chapter V.5.4). It is shown that the right translation map for each point of $T^{p}(\Gamma)$ is a biholomorphic map, which is used in the next section.

As an application of the main result, we will prove the coincidence of two canonical distances on $T^{p}(\Gamma)$ in Section 6. One is the Teichmüller distance, which is the distance proper to the Teichmüller space. The other is the Kobayashi distance, which is defined on complex manifolds and a generalization of the hyperbolic distance on 
$\Delta$. It is known that for every Fuchsian group $\Gamma$, the Teichmüller distance on $T(\Gamma)$ coincides with the Kobayashi distance, which is proved by Gardiner [10]. Earle, Gardiner and Lakic [8] proved that the Teichmüller distance coincides with the Kobayashi distance on the metric subspace of all Teichmüller equivalence classes represented by asymptotically conformal maps. Hu, Jiang and Wang [13] showed this result more directly in $T(1)$. In connection with these facts, the author [21] gave a sufficient condition for a metric subspace of $T(1)$ under which the Teichmüller distance on the subspace coincides with the Kobayashi distance. We will extend this result by using a certain exhaustion for non-compact Riemann surfaces, which is used in the triangulation of them.

\section{Characterization of $T^{p}(\Gamma)$ by Douady-Earle extensions} tion.

Throughout this paper, we always assume that $p \geq 2$ without any special men-

In this section, we characterize each point of $T^{p}(\Gamma)$ by the $p$-integrability of the Beltrami coefficient of its Douady-extension. First, let us explain about the Douady-Earle extension. Let $h$ be a sense-preserving homeomorphism of $\partial \Delta$. The Douady-Earle extension $w=E(h)(z)$ is defined by the equation

$$
F_{h}(z, w)=\frac{1}{2 \pi} \int_{\partial \Delta} \frac{h(t)-w}{1-\bar{w} h(t)} \frac{1-|z|^{2}}{|z-t|^{2}}|d t|=0 .
$$

This extension has the conformal naturality, that is, for any two Möbius transformations $g_{1}, g_{2}$ preserving $\Delta$, it follows that

$$
E\left(g_{1} \circ h \circ g_{2}\right)=g_{1} \circ E(h) \circ g_{2} .
$$

If $h$ is quasisymmetric, then $E(h)$ is a diffeomorphism and a quasiconformal selfmapping on $\Delta$. Hence for $\tau \in T(\Gamma)$ and $\mu \in \tau, E\left(\left.f^{\mu}\right|_{\partial \Delta}\right)$ is a quasiconformal self-mapping on $\Delta$. By the definition of the Teichmüller equivalence relation (1.1), the map $E$ can be regarded as the map on $T(\Gamma)$. We rewrite $E\left(\left.f^{\mu}\right|_{\partial \Delta}\right)$ as $E(\tau)$. Furthermore, the conformal naturality (2.1) implies the Beltrami coefficient $\sigma(\tau)$ of $E(\tau)$ is a $(-1,1)$-differential for $\Gamma$. Then $\sigma(\tau)$ is a representative of $\tau$. The DouadyEarle extension has an effective estimate of its Jacobian, which is used many times in this paper.

Proposition 2.1. [6, 21] Let $K \geq 1$. There exists a constant $C_{1}, C_{2}>0$ depending only on $K$ such that for every $z \in \Delta$ and every $K$-quasiconformal Douady-Earle extension $f$,

$$
\begin{aligned}
C_{1} \rho(z) & \leq(|\partial f(z)|-|\bar{\partial} f(z)|) \rho(f(z)) \\
& \leq(|\partial f(z)|+|\bar{\partial} f(z)|) \rho(f(z)) \leq C_{2} \rho(z) .
\end{aligned}
$$

Douady-Earle [6] proved this proposition first and the author [21] gave another proof. Proposition 2.1 implies that there exists a constant $C>0$ depending only on $K$ such that for any $z \in \Delta$ and any $K$-quasiconformal Douady-Earle extension,

$$
J_{f}(z) \rho(f(z))^{2} \leq C \rho(z)^{2}, \quad J_{f^{-1}}(z) \rho\left(f^{-1}(z)\right)^{2} \leq C \rho(z)^{2},
$$

where $J_{f}$ is the Jacobian of $f$. 
Before the proof of the characterization of $T^{p}(\Gamma)$, let us define some notations. For $\tau \in T(\Gamma)$ and $\mu \in \tau$, let

$$
\Gamma^{\tau}=\Gamma^{\mu}=\left\{\gamma^{\mu}=f^{\mu} \circ \gamma \circ\left(f^{\mu}\right)^{-1} \mid \gamma \in \Gamma\right\} .
$$

By the definition of the Teichmüller equivalence relation (1.1), it follows that $\Gamma^{\mu}=\Gamma^{\nu}$ for any $\mu, \nu \in \tau$, which implies $\Gamma^{\tau}$ is well-defined. Hereafter, we use these representations properly depending on the situation. Let $\mu^{-1}$ be the Beltrami coefficient of $\left(f^{\mu}\right)^{-1}$ and $\tau^{-1}$ be the point of $T\left(\Gamma^{\tau}\right)$ represented by $\mu^{-1}$.

Given $w \in \Delta$ arbitrarily. Let $\gamma^{w}(z)=(z-w) /(1-\bar{w} z)$ and $H^{w}$ be the harmonic map on $\Delta$ with boundary value $\left.\gamma^{w} \circ f^{\mu}\right|_{\partial \Delta}$ for $\mu \in \operatorname{Bel}(\Gamma)$ and

$$
\mathcal{D}\left(H^{w}\right)=\iint_{\Delta}\left(\left|\partial H^{w}(z)\right|^{2}+\left|\bar{\partial} H^{w}(z)\right|^{2}\right) d x d y
$$

be the Dirichlet integral of $H^{w}$.

As the first step of the characterization of $T^{p}(\Gamma)$, we show the following proposition.

Proposition 2.2. Let $\tau \in T(\Gamma)$ arbitrarily. If $\tau^{-1}$ belongs to $T^{p}\left(\Gamma^{\tau}\right)$, then $\sigma(\tau)^{-1}$ belongs to $\operatorname{Ael}^{p}\left(\Gamma^{\tau}\right)$.

Proof. Assume that $\tau^{-1} \in T^{p}\left(\Gamma^{\tau}\right)$. Then there exists $\nu \in \tau^{-1}$ such that $\nu$ belongs to $\operatorname{Ael}^{p}\left(\Gamma^{\tau}\right)$. It follows from the proof of Theorem 2.1 in [20] that

$$
\left(\mathcal{D}\left(H^{w}\right)-\pi\right)^{s} \rho(w)^{2} \leq \frac{C_{3}}{\pi} \iint_{\Delta}|\nu(\zeta)|^{p} \frac{d \xi d \eta}{|1-\bar{w} \zeta|^{4}}
$$

where $s=p / 2$ and $C_{3}=\left(2 \pi /\left(1-\|\nu\|_{\infty}^{2}\right)\right)^{s}$. Set $\Omega=E(\tau)(N)$. Recall that $N$ is a fundamental region on $\Delta$ for $\Gamma$. Then $\Omega$ becomes a fundamental region on $\Delta$ for $\Gamma^{\tau}$. Let $K(\zeta, w)=(1-\bar{w} \zeta)^{-4}$ be the Bergman kernel on $\Delta$. By formula (1.4) and the invariant formula of the Bergman kernel valid for all Möbius transformations, we have

$$
\begin{aligned}
\iint_{\Delta}|\nu(\zeta)|^{p}|K(\zeta, w)| d \xi d \eta & =\sum_{\gamma^{\mu} \in \Gamma^{\tau}} \iint_{\gamma^{\mu}(\Omega)}|\nu(\zeta)|^{p}|K(\zeta, w)| d \xi d \eta \\
& =\sum_{\gamma^{\mu} \in \Gamma^{\tau}} \iint_{\Omega}\left|\nu\left(\gamma^{\mu}(z)\right)\right|^{p}\left|K\left(\gamma^{\mu}(z), w\right)\right|\left|\left(\gamma^{\mu}\right)^{\prime}(z)\right|^{2} d x d y \\
& =\iint_{\Omega}|\nu(z)|^{p} \sum_{\gamma^{\mu} \in \Gamma^{\tau}}\left|K\left(z,\left(\gamma^{\mu}\right)^{-1}(w)\right)\right|\left|\left(\left(\gamma^{\mu}\right)^{-1}\right)^{\prime}(w)\right|^{2} d x d y
\end{aligned}
$$

Hence by Fubini's theorem and formula (1.4) again, we obtain that

$$
\begin{aligned}
& \iint_{\Omega}\left(\mathcal{D}\left(H^{w}\right)-\pi\right)^{s} \rho(w)^{2} d u d v \\
& \quad \leq \frac{C_{3}}{\pi} \iint_{\Omega}|\nu(z)|^{p}\left(\iint_{\Delta}|K(z, w)| d u d v\right) d x d y=C_{3}\|\nu\|_{p}^{p} .
\end{aligned}
$$

Since $\sigma(\tau) \in \operatorname{Bel}(\Gamma), \sigma(\tau)^{-1}$ belongs to $\operatorname{Bel}\left(\Gamma^{\tau}\right)$. It follows from the proof of Theorem 1 in [5] that there exists a constant $C_{4}>0$ depending only on $\|\sigma(\tau)\|_{\infty}$ such that

$$
\frac{\left|\sigma(\tau)^{-1}(w)\right|^{2}}{1-\left|\sigma(\tau)^{-1}(w)\right|^{2}} \leq C_{4}\left(\mathcal{D}\left(H^{w}\right)-\pi\right)
$$


for every $w \in \Delta$. By inequality (2.4), we obtain

$$
\begin{aligned}
\left\|\sigma(\tau)^{-1}\right\|_{p}^{p} & \leq \iint_{\Omega}\left(\frac{\left|\sigma(\tau)^{-1}(w)\right|^{2}}{1-\left|\sigma(\tau)^{-1}(w)\right|^{2}}\right)^{s} \rho(w)^{2} d u d v \\
& \leq C_{4} \iint_{\Omega}\left(\mathcal{D}\left(H^{w}\right)-\pi\right)^{s} \rho(w)^{2} d u d v \leq C_{3} C_{4}\|\nu\|_{p}^{p}<\infty .
\end{aligned}
$$

Therefore $\sigma(\tau)^{-1} \in \operatorname{Ael}^{p}\left(\Gamma^{\tau}\right)$.

The next lemma means the relation between $\sigma(\tau)$ and $\sigma(\tau)^{-1}$ in $p$-integrability.

Lemma 2.3. For every $\tau \in T(\Gamma), \sigma(\tau)$ belongs to $\operatorname{Ael}^{p}(\Gamma)$ if and only if $\sigma(\tau)^{-1}$ belongs to $\operatorname{Ael}^{p}\left(\Gamma^{\tau}\right)$.

Proof. Assume $\sigma(\tau) \in \operatorname{Ael}^{p}(\Gamma)$. By inequality (2.3), it follows that

$$
\begin{aligned}
& \iint_{E(\tau)(N)}\left|\sigma(\tau)^{-1}(w)\right|^{p} \rho(w)^{2} d u d v \\
& =\iint_{N}\left|\sigma(\tau)^{-1}(E(\tau)(z))\right|^{p} \rho(E(\tau)(z))^{2} J_{E(\tau)}(z) d x d y \\
& \leq C \iint_{N}|\sigma(\tau)(z)|^{p} \rho(z)^{2} d x d y<\infty .
\end{aligned}
$$

Thus it follows that $\sigma(\tau) \in \operatorname{Ael}^{p}(\Gamma)$. By the similar computation, the converse clearly holds.

Let us show the main result in this section.

Theorem 2.4. Let $p \geq 2$. For every $\tau \in T(\Gamma)$, the following three conditions are equivalent:

(1) $\tau \in T^{p}(\Gamma)$;

(2) $\tau^{-1} \in T^{p}\left(\Gamma^{\tau}\right)$;

(3) $\sigma(\tau) \in \operatorname{Ael}^{p}(\Gamma)$.

Proof. It is sufficient to show that condition (1) implies condition (2). Assume that condition (1) holds. Noting that $\left(\tau^{-1}\right)^{-1}=\tau, \sigma\left(\tau^{-1}\right)^{-1}$ belongs to $\operatorname{Ael}^{p}(\Gamma)$ by Proposition 2.2. It follows from Lemma 2.3 that $\sigma\left(\tau^{-1}\right)$ belongs to $\operatorname{Ael}^{p}\left(\Gamma^{\tau}\right)$. This implies that $\tau^{-1} \in T^{p}\left(\Gamma^{\tau}\right)$.

\section{Continuity of the Bers embedding}

In this section, we show the continuity of the Bers embedding $\beta$ of $T^{p}(\Gamma)$ into $A^{p}(\Gamma)$. Let us introduce a topology in $\operatorname{Ael}^{p}(\Gamma)$ by the norm $\|\cdot\|_{p, \infty}=\|\cdot\|_{p}+\|\cdot\|_{\infty}$. Define a distance on $T^{p}(\Gamma)$ as

$$
\ell_{p, \infty}(\Gamma)\left(\tau_{1}, \tau_{2}\right)=\inf \left\{\left\|\frac{\mu_{1}-\mu_{2}}{1-\mu_{1} \overline{\mu_{2}}}\right\|_{p, \infty} \mid \mu_{k} \in \varpi^{-1}\left(\tau_{k}\right) \cap \operatorname{Ael}^{p}(\Gamma)\right\}
$$

for $\tau_{k} \in T^{p}(\Gamma)(k=1,2)$. We abbreviate $\ell_{p, \infty}(\Gamma)$ as $\ell_{p, \infty}$ unless pointing out the difference between Fuchsian groups. The proof of the continuity of $\beta$ has two steps: One is that the hyperbolic $L^{p}$-norm of the Bers embedding can be estimated from above by that of $\sigma$ and the other is that the map $\sigma:\left(T^{p}(\Gamma), \ell_{p, \infty}\right) \rightarrow\left(\operatorname{Ael}^{p}(\Gamma),\|\cdot\|_{p, \infty}\right)$ is continuous. 
Let us prepare for the proof. For $\mu \in \operatorname{Bel}(\Gamma)$, we extend $\mu$ to a measurable function on $\mathbf{C}$ by setting 0 on $\overline{\Delta^{*}}$. Let $f_{\mu}$ be the quasiconformal self-mapping on $\mathbf{C}$ with extended $\mu$ satisfying

$$
\lim _{z \rightarrow \infty}\left(f_{\mu}(z)-z\right)=0 .
$$

If $f$ is a conformal map in an open neighborhood $D$ of $\infty$ and fixes $\infty$, then $f$ has a power series expansion

$$
f(z)=A z+a_{0}+\frac{a_{1}}{z}+\frac{a_{2}}{z^{2}}+\cdots
$$

for $z \in D$. Since $f_{\mu}$ is conformal in $\Delta^{*}$ and satisfies condition (3.1), $f_{\mu}$ has a series expansion

$$
f_{\mu}(z)=z+\frac{b_{1}}{z}+\frac{b_{2}}{z^{2}}+\cdots
$$

for $z \in \Delta^{*}$.

Let $S_{f_{\mu}}$ be the Schwarzian derivative of $\left.f_{\mu}\right|_{\Delta^{*}}$, that is,

$$
S_{f_{\mu}}(z)=\left(\frac{f_{\mu}^{\prime \prime}(z)}{f_{\mu}^{\prime}(z)}\right)^{\prime}-\frac{1}{2}\left(\frac{f_{\mu}^{\prime \prime}(z)}{f_{\mu}^{\prime}(z)}\right)^{2}
$$

for $z \in \Delta^{*}$. The map $\Phi: \mu \mapsto S_{f_{\mu}}$ is called the Bers projection of $\operatorname{Bel}(\Gamma)$ into $\mathcal{B}(\Gamma)$. It follows that the Teichmüller equivalence relation (1.1) is equivalent to $\left.f_{\mu}\right|_{\Delta^{*}}=$ $\left.f_{\nu}\right|_{\Delta^{*}}$. Thus $\Phi$ can be projected to the map $T(\Gamma)$ into $\mathcal{B}(\Gamma)$, which is called the Bers embedding.

Astala and Zinsmeister [2] showed an integral expression of $S_{f_{\mu}}$. We extend this result.

Lemma 3.1. For every $\mu, \nu \in \operatorname{Bel}(1)$, let $\Omega=f_{\nu}(\Delta), \Omega^{*}=f_{\nu}\left(\Delta^{*}\right)$. Define two Möbius transformations as

$$
\kappa(w)=-\frac{\left(\left|f_{\nu}^{-1}(t)\right|^{2}-1\right) f_{\mu}^{\prime}\left(f_{\nu}^{-1}(t)\right)}{w-f_{\mu}\left(f_{\nu}^{-1}(t)\right)}, \quad \lambda(w)=t-\frac{\left(\left|f_{\nu}^{-1}(t)\right|^{2}-1\right) f_{\nu}^{\prime}\left(f_{\nu}^{-1}(t)\right)}{w}
$$

for $t \in \Omega^{*}$. Then

$$
S_{f_{\mu} \circ f_{\nu}^{-1}}(t)=-\frac{6}{\pi} \rho_{\Omega^{*}}(t)^{2}\left(\frac{\left(f_{\nu}^{-1}\right)^{\prime}(t)}{\left|\left(f_{\nu}^{-1}\right)^{\prime}(t)\right|}\right)^{2} \iint_{\lambda^{-1}(\Omega)} \bar{\partial} G(\zeta) d \xi d \eta,
$$

where $G=\kappa \circ f_{\mu} \circ f_{\nu}^{-1} \circ \lambda$ and $\rho_{\Omega^{*}}=\left(\rho_{*} \circ f_{\nu}^{-1}\right)\left|\left(f_{\nu}^{-1}\right)^{\prime}\right|$ is the Poincaré metric on $\Omega^{*}$.

Proof. Let $f=f_{\mu}, g=f_{\nu}$. Since $f$ and $g$ satisfies condition (3.1), the composite map $f \circ g^{-1}$ also do so and has a series expansion of form (3.3). The function $G$ is said to be the Koebe transformation of $f \circ g^{-1}$. We claim that $G$ has a power series expansion

$$
G(z)=z+c_{0}+\frac{c_{1}}{z}+\frac{c_{2}}{z^{2}}+\cdots
$$

for $z \in \lambda^{-1}\left(\Omega^{*}\right)$. Indeed, since $G$ is conformal in $\lambda^{-1}\left(\Omega^{*}\right)$ and fixes $\infty, G$ has a power series expansion of form (3.2). From a simple computation, we have

$$
G^{\prime}(z)=\left(f \circ g^{-1}\right)^{\prime}(t)\left(f \circ g^{-1}\right)^{\prime}(w)\left(\frac{w-t}{f\left(g^{-1}(w)\right)-f\left(g^{-1}(t)\right)}\right)^{2},
$$


where $w=\lambda(z)$. Since $w$ tends to $t$ as $z$ to $\infty$, it follows that

$$
A=\lim _{z \rightarrow \infty} G^{\prime}(z)=\left(f \circ g^{-1}\right)^{\prime}(t)^{2} \cdot\left(f \circ g^{-1}\right)^{\prime}(t)^{-2}=1,
$$

and our claim holds. By Pompieu's formula, it follows that

$$
G(z)-z-c_{0}=-\frac{1}{\pi} \iint_{\lambda^{-1}(\Omega)} \frac{\bar{\partial} G(w)}{w-z} d u d v
$$

Then we have

$$
\begin{aligned}
\iint_{\lambda^{-1}(\Omega)} \bar{\partial} G(w) d u d v & =\lim _{z \rightarrow \infty} z^{2} \iint_{\lambda^{-1}(\Omega)} \frac{\bar{\partial} G(w)}{(w-z)^{2}} d u d v \\
& =-\pi \lim _{z \rightarrow \infty} z^{2}\left(G^{\prime}(z)-1\right)=\pi c_{1}=-\frac{\pi}{6} \lim _{z \rightarrow \infty} z^{4} S_{G}(z) .
\end{aligned}
$$

This implies

$$
\begin{aligned}
S_{f \circ g^{-1}}(t) & =\lim _{z \rightarrow \infty} S_{G}(z) \lambda^{\prime}(z)^{-2} \\
& =\frac{1}{\left(\left|g^{-1}(t)\right|^{2}-1\right)^{2} g^{\prime}\left(g^{-1}(t)\right)^{2}} \lim _{z \rightarrow \infty} z^{4} S_{G}(z) \\
& =-\frac{6}{\pi} \rho_{\Omega^{*}}(t)^{2}\left(\frac{\left(g^{-1}\right)^{\prime}(t)}{\left|\left(g^{-1}\right)^{\prime}(t)\right|}\right)^{2} \iint_{\lambda^{-1}(\Omega)} \bar{\partial} G(w) d u d v .
\end{aligned}
$$

Let us show the first step to the main theorem in this section.

Proposition 3.2. For every $\tau_{1}, \tau_{2} \in T^{p}(\Gamma)$, there exists a constant $C^{\prime}>0$ depending only on $\left\|\sigma\left(\tau_{1}\right)\right\|_{\infty}$ and $\left\|\sigma\left(\tau_{2}\right)\right\|_{\infty}$ such that

$$
\left\|\beta\left(\tau_{1}\right)-\beta\left(\tau_{2}\right)\right\|_{p} \leq C^{\prime}\left\|\frac{\sigma\left(\tau_{1}\right)-\sigma\left(\tau_{2}\right)}{1-\sigma\left(\tau_{1}\right) \overline{\sigma\left(\tau_{2}\right)}}\right\|_{p} .
$$

Proof. Given $\tau_{1}, \tau_{2} \in T^{p}(\Gamma)$ arbitrarily. Let $\mu=\sigma\left(\tau_{1}\right)$ and $\nu=\sigma\left(\tau_{2}\right)$. By Theorem 2.4, it follows that $\nu \in \operatorname{Ael}^{p}(\Gamma)$. Set $f=f_{\mu}, g=f_{\nu}, F=g(N), F^{*}=$ $g\left(N^{*}\right), \Omega=g(\Delta)$ and $\Omega^{*}=g\left(\Delta^{*}\right)$. Then we have

$$
\begin{aligned}
\left\|\beta\left(\tau_{1}\right)-\beta\left(\tau_{2}\right)\right\|_{p}^{p} & =\iint_{N^{*}}\left|S_{f}(z)-S_{g}(z)\right|^{p} \rho_{*}(z)^{2-2 p} d x d y \\
& =\iint_{F^{*}}\left|S_{f}\left(g^{-1}(w)\right)-S_{g}\left(g^{-1}(w)\right)\right|^{p} \rho_{*}\left(g^{-1}(w)\right)^{2-2 p}\left|\left(g^{-1}\right)^{\prime}(w)\right|^{2} d u d v \\
& =\iint_{F^{*}}\left|S_{f \circ g^{-1}}(w)\right|^{p} \rho_{\Omega^{*}}(w)^{2-2 p} d u d v
\end{aligned}
$$

where $\rho_{\Omega^{*}}=\left(\rho_{*} \circ g^{-1}\right)\left|\left(g^{-1}\right)^{\prime}\right|$ is the Poincaré metric on $\Omega^{*}$.

Take $t \in F^{*}$ arbitrarily. It follows from Lemma 3.1 that there exist two Möbius transformations $\kappa, \lambda$ such that

$$
\left|S_{f \circ g^{-1}}(t)\right|=\frac{6}{\pi} \rho_{\Omega^{*}}(t)^{2}\left|\iint_{\lambda^{-1}(\Omega)} \bar{\partial} G(\zeta) d \xi d \eta\right|,
$$


where $G=\kappa \circ f \circ g^{-1} \circ \lambda$. If we set $s=p / 2$, then by Schwarz's inequality,

$$
\begin{aligned}
\left(\frac{\pi}{6}\right)^{p}\left|S_{f \circ g^{-1}}(t)\right|^{p} \rho_{\Omega^{*}}(t)^{-2 p}=\left|\iint_{\lambda^{-1}(\Omega)} \bar{\partial} G(\zeta) d \xi d \eta\right|^{p} & \\
= & \left(\iint_{\lambda^{-1}(\Omega)} \frac{\left|\mu_{G}(\zeta)\right|^{2}}{1-\left|\mu_{G}(\zeta)\right|^{2}} d \xi d \eta\right)^{s}\left(\iint_{\lambda^{-1}(\Omega)} J_{G}(\zeta) d \xi d \eta\right)^{s}
\end{aligned}
$$

Since $G$ is conformal on $\lambda^{-1}(\Omega)$ with condition (3.1), it follows from the Area Theorem that

$$
\iint_{\lambda^{-1}(\Omega)} J_{G}(\zeta) d \xi d \eta \leq \pi
$$

By Hölder's inequality, we have

$$
\begin{aligned}
& \left(\frac{\pi}{6}\right)^{p}\left|S_{f \circ g^{-1}}(t)\right|^{p} \rho_{\Omega^{*}}(t)^{-2 p} \leq \pi^{s}\left(\iint_{\Omega} \frac{\left|\mu_{G}\left(\lambda^{-1}(w)\right)\right|^{2}}{1-\left|\mu_{G}\left(\lambda^{-1}(w)\right)\right|^{2}}\left|\left(\lambda^{-1}\right)^{\prime}(w)\right|^{2} d u d v\right)^{s} \\
& =\pi^{s}\left(\iint_{\Omega} \frac{\left|\mu_{G}\left(\lambda^{-1}(w)\right)\right|^{2}}{1-\left|\mu_{G}\left(\lambda^{-1}(w)\right)\right|^{2}} \frac{\rho_{\Omega^{*}}(t)^{-2}}{|w-t|^{4}} d u d v\right)^{s} \\
& \leq \pi^{s} \rho_{\Omega^{*}}(t)^{-p}\left(\iint_{\Omega}\left(\frac{|\alpha(w)|^{2}}{1-|\alpha(w)|^{2}}\right)^{s} \frac{d u d v}{|w-t|^{4}}\right)\left(\iint_{\Omega} \frac{d u d v}{|w-t|^{4}}\right)^{s-1}
\end{aligned}
$$

where $\alpha$ is the Beltrami coefficient of $f \circ g^{-1}$. Note that $\alpha$ is the Beltrami coefficient for $\Gamma_{g}=\left\{\gamma_{g}=g \circ \gamma \circ g^{-1} \mid \gamma \in \Gamma\right\}$. It follows that $F$ is a fundamental region on $\Omega$ for $\Gamma_{g}$. Hence $\Omega$ has a partition $\Omega=\bigcup_{\gamma_{g} \in \Gamma_{g}} \overline{\gamma_{g}(F)}$ and it follows that

$$
\iint_{\Omega} \cdots d x d y=\sum_{\gamma_{g} \in \Gamma_{g}} \iint_{\gamma_{g}(F)} \cdots d x d y
$$

Moreover, there exists the following estimate (see [17], Section 3.4.5):

$$
\iint_{\Omega} \frac{d u d v}{|w-t|^{4}} \leq 16 \pi \rho_{\Omega^{*}}(t)^{2}
$$

By the identity

$$
\frac{\gamma^{\prime}(w) \gamma^{\prime}(t)}{(\gamma(w)-\gamma(t))^{2}}=\frac{1}{(w-t)^{2}}
$$

valid for all Möbius transformations, we have

$$
\begin{aligned}
\left|S_{f \circ g^{-1}}(t)\right|^{p} \rho_{\Omega^{*}}(t)^{2-2 p} & \leq \frac{24^{p}}{16 \pi} \iint_{\Omega}\left(\frac{|\alpha(w)|^{2}}{1-|\alpha(w)|^{2}}\right)^{s} \frac{d u d v}{|w-t|^{4}} \\
& =\frac{24^{p}}{16 \pi} \sum_{\gamma_{g} \in \Gamma_{g}} \iint_{F}\left(\frac{\left|\alpha\left(\gamma_{g}(\zeta)\right)\right|^{2}}{1-\left|\alpha\left(\gamma_{g}(\zeta)\right)\right|^{2}}\right)^{s} \frac{\left|\gamma_{g}^{\prime}(\zeta)\right|^{2}}{\left|\gamma_{g}(\zeta)-t\right|^{4}} d \xi d \eta \\
& =\frac{24^{p}}{16 \pi} \iint_{F}\left(\frac{|\alpha(\zeta)|^{2}}{1-|\alpha(\zeta)|^{2}}\right)^{s}\left(\sum_{\gamma_{g} \in \Gamma_{g}} \frac{\left|\left(\gamma_{g}^{-1}\right)^{\prime}(t)\right|^{2}}{\left|\zeta-\gamma_{g}^{-1}(t)\right|^{4}}\right) d \xi d \eta
\end{aligned}
$$


Since $\Omega$ is the exterior of $\Omega^{*}$, we can change the role between $\Omega$ and $\Omega^{*}$ in formula (3.6) and (3.7). Then we have

$$
\begin{aligned}
& \left\|\beta\left(\tau_{1}\right)-\beta\left(\tau_{2}\right)\right\|_{p}^{p}=\iint_{F^{*}}\left|S_{f \circ g^{-1}}(z)\right|^{p} \rho_{\Omega^{*}}(z)^{2-2 p} d u d v \\
& \leq \frac{24^{p}}{16 \pi} \iint_{F^{*}}\left(\iint_{F}\left(\frac{|\alpha(\zeta)|^{2}}{1-|\alpha(\zeta)|^{2}}\right)^{s}\left(\sum_{\gamma_{g} \in \Gamma_{g}} \frac{\left|\left(\gamma_{g}^{-1}\right)^{\prime}(z)\right|^{2}}{\left|\zeta-\gamma_{g}^{-1}(z)\right|^{4}}\right) d \xi d \eta\right) d x d y \\
& =\frac{24^{p}}{16 \pi} \iint_{F}\left(\frac{|\alpha(\zeta)|^{2}}{1-|\alpha(\zeta)|^{2}}\right)^{s}\left(\iint_{\Omega^{*}} \frac{d x d y}{|\zeta-z|^{4}}\right) d \xi d \eta \\
& \leq 24^{p} \iint_{F}\left(\frac{|\alpha(\zeta)|^{2}}{1-|\alpha(\zeta)|^{2}}\right)^{s} \rho_{\Omega}(\zeta)^{2} d \xi d \eta \\
& =\left(\frac{24}{\sqrt{1-\|\alpha\|_{\infty}^{2}}}\right)^{p} \iint_{N}\left|\frac{\mu(w)-\nu(w)}{1-\mu(w) \overline{\nu(w)}}\right|^{p} \rho_{\Omega}(g(w))^{2} J_{g}(w)^{2} d u d v
\end{aligned}
$$

where $\rho_{\Omega}$ is the Poincaré metric on $\Omega$.

Since $g=f_{\nu}=\left(f_{\nu} \circ\left(f^{\nu}\right)^{-1}\right) \circ f^{\nu}$ and $f_{\nu} \circ\left(f^{\nu}\right)^{-1}$ is a conformal map of $\Delta$ onto $\Omega$, it follows that

By inequality (2.3), we obtain

$$
\rho_{\Omega}(g(w))^{2} J_{g}(w)=\rho\left(f^{\nu}(w)\right)^{2} J_{f^{\nu}}(w) .
$$

$$
\left\|\beta\left(\tau_{1}\right)-\beta\left(\tau_{2}\right)\right\|_{p} \leq \frac{24 C^{\frac{1}{p}}}{\sqrt{1-\|\alpha\|_{\infty}^{2}}}\left\|\frac{\mu-\nu}{1-\mu \bar{\nu}}\right\|_{p} .
$$

This proposition and Theorem 2.4 implies where $\beta\left(T^{p}(\Gamma)\right)$ is contained.

Corollary 3.3. $\beta\left(T^{p}(\Gamma)\right) \subset \beta(T(\Gamma)) \cap A^{p}(\Gamma)$.

Proof. For every $\tau \in T^{p}(\Gamma), \beta(\tau)$ belongs to $\beta(T(\Gamma))$ clearly. It is sufficient to show $\beta(\tau) \in A^{p}(\Gamma)$. By substituting $\tau_{1}=\tau$ and $\tau_{2}=0$ in inequality (3.5), it follows that

$$
\|\beta(\tau)\|_{p} \leq C^{\prime}\|\sigma(\tau)\|_{p}
$$

From Theorem 2.4, $\|\sigma(\tau)\|_{p}<\infty$ and the corollary holds.

We prepare for the proof of the continuity of $\sigma$.

Lemma 3.4. Let $\mu \in \operatorname{Bel}(\Gamma)$ and $\left\{\mu_{n}\right\}$ be a sequence of $\operatorname{Bel}(\Gamma)$ converging to $\mu$. For $0<r<1$, set $\Delta_{r}=\{|z|<r\}, N_{r}=N \backslash \bar{\Delta}_{r}, \Omega_{n, r}=E\left(\left[\mu_{n}\right]\right)\left(N_{r}\right)$. Then there exists a positive function $s_{1}(r)$ with $s_{1}(r) \rightarrow 1$ as $r \rightarrow 1$ such that

$$
t_{n}(r) \geq s_{1}(r)
$$

for all $n \geq 1$, where $t_{n}(r)$ is the Euclidean distance between 0 and

$$
F_{n, r}=\bigcup_{\gamma^{\mu_{n}} \in \Gamma^{\mu_{n}}} \overline{\gamma^{\mu_{n}}\left(\Omega_{n, r}\right)} .
$$

Proof. Let $\Omega_{r}=E([\mu])\left(N_{r}\right)$ and $t(r)$ be the Euclidean distance between 0 and $F_{r}=\bigcup_{\gamma^{\mu} \in \Gamma^{\mu}} \overline{\gamma^{\mu}\left(\Omega_{r}\right)}$. Note that both $t_{n}(r)$ and $t(r)$ are increasing functions from the interval $(0,1)$ to itself and tend to 1 as $r \rightarrow 1$. By conformal naturality (2.1), it follows that

$$
\gamma^{\mu} \circ E\left(\left.f^{\mu}\right|_{\partial \Delta}\right)=E\left(\left.f^{\mu}\right|_{\partial \Delta}\right) \circ \gamma
$$


Then we have

$$
F_{r}=\bigcup_{\gamma \in \Gamma} E([\mu])\left(\gamma\left(\bar{N}_{r}\right)\right) .
$$

By the similar computation, the equality

$$
F_{n, r}=\bigcup_{\gamma^{\mu_{n}} \in \Gamma^{\mu_{n}}} E\left(\left[\mu_{n}\right]\right)\left(\gamma\left(\bar{N}_{r}\right)\right)
$$

also holds. These expressions imply that there exist $w, w_{n} \in \bar{N}_{r} \cap \partial \Delta_{r}$ and $\gamma, \gamma_{n} \in \Gamma$ such that

$$
|E([\mu])(\gamma(w))|=t(r),\left|E\left(\left[\mu_{n}\right]\right)\left(\gamma_{n}\left(w_{n}\right)\right)\right|=t_{n}(r),
$$

respectively. By taking a subsequence, we can assume that either $t_{n}(r) \geq t(r)$ or $t_{n}(r) \leq t(r)$ holds for all $n \geq 1$. When $t_{n}(r) \geq t(r)$, it follows from the definition of $t_{n}(r)$ that

$0 \leq t_{n}(r)-t(r) \leq\left|E\left(\left[\mu_{n}\right]\right)(\gamma(w))\right|-|E([\mu])(\gamma(w))| \leq \sup _{w \in \Delta}\left|E\left(\left[\mu_{n}\right]\right)(w)-E([\mu])(w)\right|$.

By the similar method, the inequality

$$
0 \leq t(r)-t_{n}(r) \leq \sup _{w \in \Delta}\left|E\left(\left[\mu_{n}\right]\right)(w)-E([\mu])(w)\right|
$$

holds when $t_{n}(r) \leq t(r)$. Then we have

$$
\sup _{r \in(0,1)}\left|t_{n}(r)-t(r)\right| \leq \sup _{w \in \Delta}\left|E\left(\left[\mu_{n}\right]\right)(w)-E([\mu])(w)\right| .
$$

Since $E\left(\left[\mu_{n}\right]\right)$ converges to $E([\mu])$ uniformly on $\Delta$ as $n \rightarrow \infty$,

$$
t_{n}(r) \rightarrow t(r)
$$

uniformly in $r$ as $n \rightarrow \infty$. Let us show $t_{n}(r)$ converges to 1 as $r \rightarrow 1$ and $n \rightarrow \infty$. Take $\varepsilon>0$ arbitrarily. Since $t(r)$ converges to 1 as $r \rightarrow 1$, there exists a real number $r_{0} \in(0,1)$ such that $1-t(r)<\frac{\varepsilon}{2}$ for $r \geq r_{0}$. By the result (3.8), there exists a number $n_{0} \in \mathbf{N}$ such that $\sup _{r \in(0,1)}\left|t_{n}(r)-t(r)\right|<\frac{\varepsilon}{2}$ for $n \geq n_{0}$. Hence we have

$$
1-t_{n}(r) \leq 1-t(r)+\sup _{r \in(0,1)}\left|t_{n}(r)-t(r)\right|<\varepsilon
$$

for $r \geq r_{0}$ and $n \geq n_{0}$. This implies that

$$
t_{n}(r) \rightarrow 1
$$

as $r \rightarrow 1$ and $n \rightarrow \infty$.

Set

$$
s_{1}(r)=t(r)-\sup _{n \in \mathbf{N}}\left|t_{n}(r)-t(r)\right|
$$

We prove that $s_{1}$ is the desired function. It clearly follows that $s_{1}(r) \leq t_{n}(r)$ for all $n \geq 1$, and it is sufficient to show that $\sup _{n \in \mathbf{N}}\left|t_{n}(r)-t(r)\right|$ converges to 0 as $r \rightarrow 1$. For any $\varepsilon>0$, it follows from the definition of $t(r)$ and the result (3.9) that there exist numbers $r_{0}^{\prime} \in(0,1)$ and $n_{0}^{\prime} \in \mathbf{N}$ such that

$$
1-t(r)<\frac{\varepsilon}{2}, 1-t_{n}(r)<\frac{\varepsilon}{2}
$$

for $r \geq r_{0}^{\prime}$ and $n \geq n_{0}^{\prime}$. Then we have

$$
\left|t_{n}(r)-t(r)\right| \leq(1-t(r))+\left(1-t_{n}(r)\right)<\varepsilon
$$


for $r \geq r_{0}^{\prime}$ and $n \geq n_{0}^{\prime}$. By taking a subsequence, we can assume that the inequality (3.10) holds for all $n \geq 1$. Hence $\sup _{n \in \mathbf{N}}\left|t_{n}(r)-t(r)\right|$ converges to 0 as $r \rightarrow 0$ and the lemma follows.

Now let us show the continuity of $\sigma$.

Proposition 3.5. The map $\sigma$ is a continuous map of $\left(T^{p}(\Gamma), \ell_{p, \infty}\right)$ into $\left(\operatorname{Ael}^{p}(\Gamma)\right.$, $\left.\|\cdot\|_{p, \infty}\right)$.

Proof. We use the notations in Lemma 3.4. Let $\tilde{\sigma}=\sigma \circ \varpi$. Recall that $\varpi$ is the quotient map of $\operatorname{Bel}(\Gamma)$ onto $T(\Gamma)$. Since $\ell_{p, \infty}$ is the quotient distance induced by $\|\cdot\|_{p, \infty}$, it is sufficient to show that $\tilde{\sigma}$ is a continuous map of $\left(\operatorname{Ael}^{p}(\Gamma),\|\cdot\|_{p, \infty}\right)$ into itself.

Take $\mu \in \operatorname{Ael}^{p}(\Gamma)$ arbitrarily. Let $0<k<1, k^{\prime}>0$ with

$$
\|\mu\|_{\infty}<k,\|\mu\|_{p}<k^{\prime}
$$

respectively, and let $\left\{\mu_{n}\right\}$ be a sequence of $\operatorname{Ael}^{p}(\Gamma)$ converging to $\mu$ in $\operatorname{Ael}^{p}(\Gamma)$. Since

$$
\left\|\mu_{n}\right\|_{\infty}<k,\left\|\mu_{n}\right\|_{p}<k^{\prime}
$$

for sufficiently large $n$, we can assume that this condition holds for all $n \geq 1$.

For $0<r<1$, set

$$
\varepsilon_{\mu}(r)=\iint_{N_{r}}|\mu(z)|^{p} \rho(z)^{2} d x d y
$$

Before we show the continuity of $\tilde{\sigma}$, we estimate $\varepsilon_{\tilde{\sigma}\left(\mu_{n}\right)}(r)$. By inequality (2.3), it follows that

$$
\varepsilon_{\tilde{\sigma}\left(\mu_{n}\right)}(r) \leq C \iint_{\Omega_{n, r}}\left|\tilde{\sigma}\left(\mu_{n}\right)^{-1}(w)\right|^{p} \rho(w)^{2} d u d v
$$

where $C$ is a constant depending only on $k$. Set $\nu_{n}=\tilde{\sigma}\left(\mu_{n}^{-1}\right)^{-1}$. Noting that $\nu_{n}$ is Teichmüller equivalent to $\mu_{n}$, we have $\Gamma^{\nu_{n}}=\Gamma^{\mu_{n}}$ and $\tilde{\sigma}\left(\mu_{n}\right)=\tilde{\sigma}\left(\nu_{n}\right)$. From the proof of Theorem 2.4, there exists a constant $C_{5}>0$ depending only on $k$ such that

$$
\iint_{\Omega_{n, r}}\left|\tilde{\sigma}\left(\nu_{n}\right)^{-1}(w)\right|^{p} \rho(w)^{2} d u d v \leq C_{5} \iint_{\Omega_{n, r}}\left(\iint_{\Delta}\left(\frac{\left|\nu_{n}^{-1}(z)\right|^{2}}{1-\left|\nu_{n}^{-1}(z)\right|^{2}}\right)^{s} \frac{d x d y}{|1-\bar{w} z|^{4}}\right) d u d v
$$

where $s=p / 2$. Let $\Omega_{n}=E\left(\left[\mu_{n}\right]\right)(N)$. Since $\Omega_{n}$ is a fundamental region for $\Gamma^{\mu_{n}}=$ $\Gamma^{\nu_{n}}$, it follows from the similar computation in the proof of Theorem 2.4 that

$$
\begin{aligned}
& \iint_{\Omega_{n, r}}\left(\iint_{\Delta}\left(\frac{\left|\nu_{n}^{-1}(z)\right|^{2}}{1-\left|\nu_{n}^{-1}(z)\right|^{2}}\right)^{s} \frac{d x d y}{|1-\bar{w} z|^{4}}\right) d u d v \\
& =\iint_{\Omega_{n}}\left(\frac{\left|\nu_{n}^{-1}(\zeta)\right|^{2}}{1-\left|\nu_{n}^{-1}(\zeta)\right|^{2}}\right)^{s}\left(\iint_{F_{n, r}} \frac{d x d y}{|1-\bar{z} \zeta|^{4}}\right) d \xi d \eta \\
& \leq \frac{1}{\left(1-\left\|\nu_{n}^{-1}\right\|_{\infty}^{2}\right)^{s}} \iint_{\Omega_{n}}\left|\nu_{n}^{-1}(\zeta)\right|^{p}\left(\iint_{\Delta \backslash \bar{\Delta}_{t_{n}(r)}} \frac{d x d y}{|1-\bar{z} \zeta|^{4}}\right) d \xi d \eta \\
& =\frac{1}{\left(1-\left\|\nu_{n}^{-1}\right\|_{\infty}^{2}\right)^{s}} \iint_{\Omega_{n}}\left|\nu_{n}^{-1}(\zeta)\right|^{p} \frac{\left(1-t_{n}(r)^{2}\right)\left(1-t_{n}(r)^{2}|\zeta|^{4}\right)}{\left(1-t_{n}(r)^{2}|\zeta|^{2}\right)^{2}} \rho(\zeta)^{2} d \xi d \eta
\end{aligned}
$$

Here the last equality follows from the identity

$$
\iint_{\Delta \backslash \bar{\Delta}_{r}} \frac{d x d y}{|1-\bar{z} \zeta|^{4}}=\frac{\left(1-r^{2}\right)\left(1-r^{2}|\zeta|^{4}\right)}{\left(1-r^{2}|\zeta|^{2}\right)^{2}} \rho(\zeta)^{2}
$$


which holds by Parseval's identity. We divide the domain $\Omega_{n}$ of integration into $\Omega_{n, r^{\prime}}$ and $\Omega_{n} \backslash \Omega_{n, r^{\prime}}=E\left(\left[\mu_{n}\right]\right)\left(N \cap \bar{\Delta}_{r^{\prime}}\right)$. The first term implies that

$$
\iint_{\Omega_{n, r^{\prime}}}\left|\nu_{n}^{-1}(\zeta)\right|^{p} \frac{\left(1-t_{n}(r)^{2}\right)\left(1-t_{n}(r)^{2}|\zeta|^{4}\right)}{\left(1-t_{n}(r)^{2}|\zeta|^{2}\right)^{2}} \rho(\zeta)^{2} d \xi d \eta \leq \iint_{\Omega_{n}}\left|\nu_{n}^{-1}(\zeta)\right|^{p} \rho(\zeta)^{2} d \xi d \eta .
$$

Set the last term as $\varepsilon_{\nu_{n}^{-1}}\left(r^{\prime}\right)$. On the other hand, it follows from Lemma 3.4 that

$$
\begin{aligned}
& \iint_{\Omega_{n} \backslash \Omega_{n, r^{\prime}}}\left|\nu_{n}^{-1}(\zeta)\right|^{p} \frac{\left(1-t_{n}(r)^{2}\right)\left(1-t_{n}(r)^{2}|\zeta|^{4}\right)}{\left(1-t_{n}(r)^{2}|\zeta|^{2}\right)^{2}} \rho(\zeta)^{2} d \xi d \eta \\
& \leq\left(1-s_{1}(r)^{2}\right) \iint_{\Omega_{n}} \frac{1+|\zeta|^{2}}{1-|\zeta|^{2}}\left|\nu_{n}^{-1}(\zeta)\right|^{p} \rho(\zeta)^{2} d \xi d \eta \leq \frac{1+M_{n}\left(r^{\prime}\right)^{2}}{1-M_{n}\left(r^{\prime}\right)^{2}}\left\|\nu_{n}^{-1}\right\|_{p}^{p}\left(1-s_{1}(r)^{2}\right),
\end{aligned}
$$

where

$$
M_{n}\left(r^{\prime}\right)=\max \left\{|z| \mid z \in E\left(\left[\mu_{n}\right]\right)\left(N \cap \partial \Delta_{r^{\prime}}\right)\right\} .
$$

Set

$$
M\left(r^{\prime}\right)=\max \left\{|z| \mid z \in E([\mu])\left(N \cap \partial \Delta_{r^{\prime}}\right)\right\} .
$$

By the similar argument to the proof of Lemma 3.4, the function

$$
L\left(r^{\prime}\right)=M\left(r^{\prime}\right)+\sup _{n \in \mathbf{N}}\left|M_{n}\left(r^{\prime}\right)-M\left(r^{\prime}\right)\right|
$$

is less than 1 for the real number $r^{\prime} \in(0,1)$ sufficiently close to 1 and

$$
M_{n}\left(r^{\prime}\right) \leq L\left(r^{\prime}\right)
$$

for $n \geq 1$. By Proposition 7 in [6], there exists a constant $0<c<1$ depending only on $k$ such that

$$
\left\|\nu_{n}^{-1}\right\|_{\infty}=\left\|\tilde{\sigma}\left(\mu_{n}^{-1}\right)\right\|_{\infty}<c
$$

Moreover it follows from the proof of Proposition 2.2 that there exists a constant $C_{6}>0$ depending only on $k$ such that

$$
\left\|\nu_{n}^{-1}\right\|_{p} \leq C_{6}\left\|\mu_{n}\right\|_{p}
$$

By inequalities (3.11), (3.13), (3.14), and (3.15), we have

$$
\begin{aligned}
& \iint_{\Omega_{n, r}}\left|\tilde{\sigma}\left(\mu_{n}^{-1}\right)(w)\right|^{p} \rho(w)^{2} d u d v \\
& <\frac{C_{5}}{\left(1-c^{2}\right)^{s}}\left(\varepsilon_{\tilde{\sigma}\left(\mu_{n}^{-1}\right)}\left(r^{\prime}\right)+\frac{1+L\left(r^{\prime}\right)^{2}}{1-L\left(r^{\prime}\right)^{2}} C_{6}^{p} k^{\prime p}\left(1-s_{1}(r)^{2}\right)\right) .
\end{aligned}
$$

Let us estimate $\varepsilon_{\tilde{\sigma}\left(\mu_{n}^{-1}\right)}\left(r^{\prime}\right)$. By using inequality (2.3) again, it follows that

$$
\varepsilon_{\tilde{\sigma}\left(\mu_{n}^{-1}\right)}\left(r^{\prime}\right) \leq C \iint_{N_{r^{\prime}}}\left|\tilde{\sigma}\left(\mu_{n}^{-1}\right)^{-1}(w)\right|^{p} \rho(w)^{2} d u d v
$$

Similarly to the way to obtain inequality (3.16), we have

$$
\begin{aligned}
& \iint_{N_{r^{\prime}}}\left|\tilde{\sigma}\left(\mu_{n}^{-1}\right)^{-1}(w)\right|^{p} \rho(w)^{2} d u d v \\
& \leq \frac{C_{5}}{\left(1-c^{2}\right)^{s}}\left(\varepsilon_{\mu_{n}}\left(r^{\prime \prime}\right)+\frac{1+r^{\prime \prime 2}}{1-r^{\prime \prime 2}} k^{\prime p}\left(1-s_{2}\left(r^{\prime}\right)^{2}\right)\right),
\end{aligned}
$$


where $r^{\prime \prime} \in(0,1)$ and $s_{2}\left(r^{\prime}\right)$ be the distance between 0 and $\bigcup_{\gamma \in \Gamma} \gamma\left(N_{r^{\prime}}\right)$. Finally, we estimate $\varepsilon_{\mu_{n}}\left(r^{\prime \prime}\right)$.

$$
\begin{aligned}
\varepsilon_{\mu_{n}}\left(r^{\prime \prime}\right) & \leq \iint_{N_{r^{\prime \prime}}}\left(|\mu(z)|+\left|\mu_{n}(z)-\mu(z)\right|\right)^{p} \rho(z)^{2} d x d y \\
& \leq 2^{p-1}\left(\varepsilon_{\mu}\left(r^{\prime \prime}\right)+\left\|\mu_{n}-\mu\right\|_{p}^{p}\right) .
\end{aligned}
$$

Now let us show the continuity of $\tilde{\sigma}$. Since $\tilde{\sigma}$ is continuous from $\left(\operatorname{Bel}(\Gamma),\|\cdot\|_{\infty}\right)$ into itself (see [6]), it is sufficient to prove $\tilde{\sigma}\left(\mu_{n}\right)$ converges to $\tilde{\sigma}(\mu)$ in hyperbolic $L^{p}$-norm. For every $\varepsilon^{\prime}>0$, take $r_{1} \in(0,1)$ with

$$
\frac{2^{p-1} C C_{5}}{\left(1-k^{2}\right)^{s}} \varepsilon_{\mu}\left(r_{1}\right)<\frac{\varepsilon^{\prime}}{2}
$$

For such $r_{1}$, there exists a real number $r_{2} \in(0,1)$ such that

$$
\frac{C C_{5}}{\left(1-k^{2}\right)^{s}} \frac{1+r_{1}^{2}}{1-r_{1}^{2}} k^{\prime p}\left(1-s_{2}\left(r_{2}\right)^{2}\right)<\frac{\varepsilon^{\prime}}{2} \text {. }
$$

Then it follows from (3.17), (3.18) and (3.19) that

$$
\begin{aligned}
\varepsilon_{\tilde{\sigma}\left(\mu_{n}^{-1}\right)}\left(r_{2}\right) & \leq \frac{C C_{5}}{\left(1-k^{2}\right)^{s}}\left(\varepsilon_{\mu_{n}}\left(r_{1}\right)+\frac{1+r_{1}^{2}}{1-r_{1}^{2}} k^{\prime p}\left(1-s_{2}\left(r_{2}\right)^{2}\right)\right) \\
& <\varepsilon^{\prime}+\frac{C C_{5}}{\left(1-k^{2}\right)^{s}}\left\|\mu_{n}-\mu\right\|_{p}^{p} .
\end{aligned}
$$

Set $\varepsilon=\left(2^{p+2} C C_{5} \varepsilon^{\prime}\right) /\left(1-c^{2}\right)^{s}$. For such $\varepsilon$, there exists a real number $r_{3} \in(0,1)$ such that

$$
\frac{C C_{5}}{\left(1-c^{2}\right)^{s}} \frac{1+L\left(r_{2}\right)^{2}}{1-L\left(r_{2}\right)^{2}} C_{6}^{p} k^{\prime p}\left(1-s_{1}(r)^{2}\right)<\frac{\varepsilon}{2^{p+2}},
$$

for $r \geq r_{3}$. By (3.12) and (3.16), we have

$$
\begin{aligned}
\varepsilon_{\tilde{\sigma}\left(\mu_{n}\right)}(r) & \leq \frac{C C_{5}}{\left(1-c^{2}\right)^{s}}\left(\varepsilon_{\tilde{\sigma}\left(\mu_{n}^{-1}\right)}\left(r_{2}\right)+\frac{1+L\left(r_{2}\right)^{2}}{1-L\left(r_{2}\right)^{2}} C_{6}^{p} k^{\prime p}\left(1-s_{1}(r)^{2}\right)\right) \\
& <\frac{\varepsilon}{2^{p+1}}+\frac{C^{2} C_{5}^{2}}{\left(1-k^{2}\right)^{s}\left(1-c^{2}\right)^{s}}\left\|\mu_{n}-\mu\right\|_{p}^{p} .
\end{aligned}
$$

By the similar method, there exists a real number $r_{4} \in(0,1)$ such that

$$
\varepsilon_{\tilde{\sigma}(\mu)}(r)<\frac{\varepsilon}{2^{p+1}}
$$

for $r \geq r_{4}$. Take $\max \left\{r_{3}, r_{4}\right\} \leq r_{0}<1$. Recall that $\mu_{n}$ converges $\mu$ in $\|\cdot\|_{p, \infty}$ and that $\tilde{\sigma}$ is continuous in $\|\cdot\|_{\infty}$. Hence for such $r_{0}$, there exists a number $n_{r_{0}} \in \mathbf{N}$ such that

$$
\operatorname{Area}\left(N \cap \Delta_{r_{0}}\right)\left\|\tilde{\sigma}\left(\mu_{n}\right)-\tilde{\sigma}(\mu)\right\|_{\infty}^{p}<\frac{\varepsilon}{4}
$$

for $n \geq n_{r_{0}}$, where $\operatorname{Area}\left(N \cap \Delta_{r_{0}}\right)$ is the hyperbolic area of $N \cap \Delta_{r_{0}}$. On the other hand, there exists a number $n^{\prime} \in \mathbf{N}$ such that

$$
\frac{2^{p-1} C^{2} C_{5}^{2}}{\left(1-k^{2}\right)^{s}\left(1-c^{2}\right)^{s}}\left\|\mu_{n}-\mu\right\|_{p}^{p}<\frac{\varepsilon}{4} .
$$


for $n \geq n^{\prime}$. Set $n_{0}=\max \left\{n_{r_{0}}, n^{\prime}\right\}$. Therefore we obtain

$$
\begin{aligned}
\| \tilde{\sigma}\left(\mu_{n}\right) & -\tilde{\sigma}(\mu) \|_{p}^{p}=\left(\iint_{N_{r_{0}}}+\iint_{N \cap \Delta_{r_{0}}}\right)\left|\tilde{\sigma}\left(\mu_{n}\right)(z)-\tilde{\sigma}(\mu)(z)\right|^{p} \rho(z)^{2} d x d y \\
& \leq 2^{p-1}\left(\varepsilon_{\tilde{\sigma}\left(\mu_{n}\right)}\left(r_{0}\right)+\varepsilon_{\tilde{\sigma}(\mu)}\left(r_{0}\right)\right)+\operatorname{Area}\left(N \cap \Delta_{r_{0}}\right)\left\|\tilde{\sigma}\left(\mu_{n}\right)-\tilde{\sigma}(\mu)\right\|_{\infty}^{p} \\
& <2^{p-1}\left(\frac{\varepsilon}{2^{p}}+\frac{C^{2} C_{5}^{2}}{\left(1-k^{2}\right)^{s}\left(1-c^{2}\right)^{s}}\left\|\mu_{n}-\mu\right\|_{p}^{p}\right)+\frac{\varepsilon}{4}<\varepsilon .
\end{aligned}
$$

for $n \geq n_{0}$. Since $\varepsilon$ is taken arbitrarily, the proposition holds.

By Proposition 3.2 and 3.5, we obtain the main result in this section:

Proposition 3.6. Let $p \geq 2$. Then the Bers embedding is a continuous map of $\left(T^{p}(\Gamma), \ell_{p, \infty}\right)$ into $\left(A^{p}(\Gamma),\|\cdot\|_{p}\right)$.

This proposition implies that the Bers projection $\Phi: \operatorname{Ael}^{p}(\Gamma) \rightarrow A^{p}(\Gamma)$ is a continuous map.

Remark. Recall that 1 is the trivial group. For every Fuchsian group $\Gamma, \operatorname{Bel}(\Gamma)$ and $\mathcal{B}(\Gamma)$ are contained in $\operatorname{Bel}(1)$ and $\mathcal{B}(1)$, respectively. By this fact, we can apply the proof of the continuity of the Bers embedding on the universal Teichmüller space $T(1)$ to the case of $T(\Gamma)$ (see Theorem V.4.8 in [16]). However, we cannot take the similar way for $p$-integrable Teichmüller spaces in general. Indeed, $\operatorname{Ael}^{p}(\Gamma)$ and $A^{p}(\Gamma)$ are contained in $\operatorname{Ael}^{p}(1)$ and $A^{p}(1)$, respectively if and only if $\Gamma$ is a finite group. Hence Proposition 3.6 is a non-trivial result for every Fuchsian group with infinitely many elements.

\section{Continuity of the inverse map of the Bers embedding}

In this section, we prove the continuity of the inverse map of the Bers embedding. Note that some results in this section holds for $p \geq 1$. We apply the argument in the case of the whole space $T(\Gamma)$. In other words, for every $\varphi \in \beta(T(\Gamma))$, there exist a open neighborhood $U_{\varphi}$ of $\varphi$ and a continuous map $s_{\varphi}$ of $U_{\varphi}$ into $\operatorname{Bel}(\Gamma)$ such that $\beta^{-1}=\varpi \circ s_{\varphi}$ on $U_{\varphi}$ (see Chapter III.4.2 in [16]). In the proof, the following result is used:

Proposition 4.1. [1] Let $A$ be a $K$-quasidisc and $\rho_{A}$ be the Poincaré metric on $A$. Then there exists a constant $\varepsilon>0$ depending only on $K$, such that every meromorphic function $f$ in $A$ with the property

$$
\left\|S_{f}\right\|_{A}=\sup _{z \in A}\left|S_{f}(z)\right| \rho_{A}(z)^{-2} \leq \varepsilon
$$

is univalent in $A$ and can be extended to a quasiconformal mapping of $\mathbf{C}$ whose complex dilatation $\mu$ satisfies the inequality

$$
|\mu(w)| \leq \frac{1}{\varepsilon}\left|S_{f}(\psi(w))\right| \rho_{A}(\psi(w))^{-2} \quad(w \in \hat{\mathbf{C}} \backslash A) .
$$

Here $\psi$ is a quasiconformal reflection in $\partial A$, continuously differentiable in $A$ and $\hat{\mathbf{C}} \backslash \bar{A}$.

Let us explain about some notations in this proposition. A $K$-quasidisc is the image of $\Delta$ by a $K$-quasiconformal mapping. Let $c$ be a Jordan curve bounding the domains $A_{1}$ and $A_{2}$. A sense-reversing quasiconformal involution of the extended 
complex plane $\hat{\mathbf{C}}$ which maps $A_{1}$ onto $A_{2}$ is a quasiconformal reflection in $c$ if it keeps every point of $c$ fixed. From [1], for every $K$-quasidisc $A$, there exist constants $c_{1}, c_{2}>$ 0 depending only on $K$ and a $c_{1}$-quasiconformal reflection $\psi$ in $\partial A$, continuously differentiable in $A$ and $\hat{\mathbf{C}} \backslash \bar{A}$, such that

$$
\left|\frac{d \psi(z)}{d z}\right| \leq c_{2}
$$

at every point $z \in A$. This fact is used in the proof of Proposition 4.1.

The next proposition means that we can compare two norms $\|\cdot\|_{p}$ and $\|\cdot\|_{\infty}$ under a geometric condition for $\Gamma$.

Proposition 4.2. Let $p \geq 1$ and $\Gamma$ be a Fuchsian group acting on $\Delta$. If the infimum $\ell$ of the lengths of all simple closed geodesics in the Riemann surface $\Delta / \Gamma$ is positive, then there exists a constant $C_{p}=C_{p}(\ell)>0$ depending on $p$ and $\ell$ such that

$$
\|\varphi\|_{\infty} \leq C_{p}\|\varphi\|_{p}
$$

for all $\varphi \in A^{p}(\Gamma)$. Especially, $A^{p}(\Gamma) \subset \mathcal{B}(\Gamma)$.

Proof. By Theorem 1 in [15], it follows that inequality (4.2) holds for $p=1$. The main theorem in [19] says that if inequality (4.2) holds for some $p \geq 1$, then the inequality also holds for every $p \geq 1$. Hence the proposition follows.

Let us call this geometric condition for $\Gamma$ Lehner's condition. We list some examples of Fuchsian groups with respect to Lehner's condition.

(1) If $\Gamma$ is a lattice, that is, if some Dirichlet region for $\Gamma$ has a finite hyperbolic area, then $\Gamma$ satisfies Lehner's condition clearly. In this case, $A^{p}(\Gamma)$ coincides with $\mathcal{B}(\Gamma)$.

(2) If $\Gamma$ is a finitely generated Fuchsian group, then $\Gamma$ satisfies Lehner's condition. Indeed, the number of hyperbolic generators in $\Gamma$ is finite. Then there exists a minimum of the traces of such elements, and its value is greater than 2 .

(3) If $\Gamma$ is an infinitely generated Fuchsian group and if the infimum of the traces of hyperbolic generators in $\Gamma$ is 2 , then $\Gamma$ does not satisfy Lehner's condition.

In fact, since Niebur and Sheingorn [18] proved the converse of Theorem 1 in [15], we see that Lehner's condition is equivalent to condition (4.2).

Now let us show the continuity of the inverse map $\beta^{-1}$.

Proposition 4.3. Let $p \geq 1$. If a Fuchsian group $\Gamma$ satisfies Lehner's condition, then the inverse map of the Bers embedding is a continuous map of $\left(\beta\left(T^{p}(\Gamma)\right),\|\cdot\|_{p}\right)$ onto $\left(T^{p}(\Gamma), \ell_{p, \infty}\right)$.

Proof. Take $\mu \in \operatorname{Ael}^{p}(\Gamma)$ arbitrarily. Let $D_{p}(\beta([\mu]), r)=\left\{\varphi \in A^{p}(\Gamma) \mid \| \varphi-\right.$ $\left.\beta([\mu]) \|_{p}<r\right\}$ for $r>0$. It follows from Proposition 4.2 that there exists a constant $C_{p}>0$ such that

$$
\|\varphi-\beta([\mu])\|_{\infty} \leq C_{p}\|\varphi-\beta([\mu])\|_{p}<C_{p} r
$$

for any $\varphi \in D_{p}(\beta([\mu]), r)$. Since $\varphi$ is a holomorphic function on $\Delta^{*}$, there exists a meromorphic function $h$ such that $S_{h}=\varphi$ (cf. [16], Theorem II.1.1). Then we have $\|\varphi-\beta([\mu])\|_{\infty}=\left\|S_{h \circ f_{\mu}^{-1}}\right\|_{\infty}$. Set $g=h \circ f_{\mu}^{-1}$.

Let $K([\mu])$ be the smallest maximal dilatation of all representatives in $[\mu]$. Since $f_{\mu}\left(\Delta^{*}\right)$ is $K([\mu])$-quasidisc, it follows from Proposition 4.1 that there exists a constant 
$\varepsilon>0$ depending only on $K([\mu])$ such that every meromorphic function $f$ on $f_{\mu}\left(\Delta^{*}\right)$ satisfying $\left\|S_{f}\right\|_{\infty}<\varepsilon$ is univalent in $f_{\mu}\left(\Delta^{*}\right)$ and has a quasiconformal extension to the complex plane. By taking $r=\varepsilon / C_{p}$, it follows that $\left\|S_{g}\right\|_{\infty}=\|\varphi-\beta([\mu])\|_{\infty}<\varepsilon$. Hence $g$ is univalent in $f_{\mu}\left(\Delta^{*}\right)$ and has a quasiconformal extension to the complex plane, denoted by $\tilde{g}$. If we set $\tilde{h}=\tilde{g} \circ f_{\mu}$, then $\tilde{h}$ is quasiconformal on $\Delta$ and coincides with $h$ on $\Delta^{*}$. Then $\varphi=S_{\tilde{h}}$ belongs to $\beta(T(\Gamma))$. This implies $D_{p}(\beta([\mu]), r) \subset$ $\beta(T(\Gamma)) \cap A^{p}(\Gamma)$.

Let $\delta$ and $\omega$ be the Beltrami coefficients of $\tilde{g}$ and $\tilde{h}$, respectively. By Proposition $4.1, \delta$ has the following estimate:

$$
|\delta(\zeta)| \leq \frac{1}{\varepsilon}\left|S_{g}(\psi(\zeta))\right| \rho_{f_{\mu}\left(\Delta^{*}\right)}(\psi(\zeta))^{-2}
$$

for $\zeta \in f_{\mu}(\Delta)$, where $\psi$ is a quasiconformal reflection in $\partial f_{\mu}(\Delta)$, continuously differentiable in $f_{\mu}(\Delta)$ and $\hat{\mathbf{C}} \backslash \overline{f_{\mu}(\Delta)}$. If we set $\zeta=f_{\mu}(z)$, then it follows that

$$
\left|\delta\left(f_{\mu}(z)\right)\right|=\left|\frac{\omega(z)-\mu(z)}{1-\omega(z) \overline{\mu(z)}}\right| .
$$

This formula and inequality (4.3) imply that

$$
\begin{aligned}
\left\|\frac{\omega-\mu}{1-\omega \bar{\mu}}\right\|_{p}^{p} & =\iint_{N}\left|\frac{\omega(z)-\mu(z)}{1-\omega(z) \overline{\mu(z)}}\right|^{p} \rho(z)^{2} d x d y \\
& \leq \iint_{N} \frac{1}{\varepsilon^{p}}\left|S_{g}\left(f_{\mu}\left(\bar{z}^{-1}\right)\right)\right|^{p} \rho_{f_{\mu}\left(\Delta^{*}\right)}\left(f_{\mu}\left(\bar{z}^{-1}\right)\right)^{-2 p} \rho(z)^{2} d x d y \\
& =\iint_{N^{*}} \frac{1}{\varepsilon^{p}}\left|S_{g}\left(f_{\mu}(w)\right)\right|^{p} \rho_{f_{\mu}\left(\Delta^{*}\right)}\left(f_{\mu}(w)\right)^{-2 p} \rho\left(\bar{w}^{-1}\right)^{2} \frac{d u d v}{|w|^{2}}
\end{aligned}
$$

Noting that $S_{g}=\left(S_{f_{\omega}} \circ f_{\mu}^{-1}-S_{f_{\mu}} \circ f_{\mu}^{-1}\right)\left(\left(f_{\mu}^{-1}\right)^{\prime}\right)^{2}$ and $\rho_{f_{\mu}\left(\Delta^{*}\right)}=\left(\rho_{*} \circ f_{\mu}^{-1}\right)\left|\left(f_{\mu}^{-1}\right)^{\prime}\right|$, we have

$$
\left\|\frac{\omega-\mu}{1-\omega \bar{\mu}}\right\|_{p}^{p} \leq \frac{1}{\varepsilon^{p}} \iint_{N^{*}}\left|S_{f_{\omega}}(w)-S_{f_{\mu}}(w)\right|^{p} \rho_{*}(w)^{2-2 p} d u d v=\left(\frac{\|\beta([\omega])-\beta([\mu])\|_{p}}{\varepsilon}\right)^{p} .
$$

This implies clearly that $\omega \in \operatorname{Ael}^{p}(\Gamma)$. Recall that $\varepsilon$ is depending only on $K([\mu])$. Since $\ell_{p, \infty}$ is the quotient distance induced by $\|\cdot\|_{p, \infty},[\omega]$ converges to $[\mu]$ in $\ell_{p, \infty}$ as $\beta([\omega]) \rightarrow \beta([\mu])$ in $\|\cdot\|_{p}$ and the inverse map of $\beta$ is continuous.

From Proposition 3.6 and 4.3, we obtain the main result of this paper.

Theorem 4.4. Let $p \geq 2$. If a Fuchsian group $\Gamma$ satisfies Lehner's condition, then the Bers embedding is a homeomorphism of $\left(T^{p}(\Gamma), \ell_{p, \infty}\right)$ into $\left(A^{p}(\Gamma),\|\cdot\|_{p}\right)$.

\section{Biholomorphically equivalent complex structure}

In this section, we introduce a biholomorphic map between $p$-integrable Teichmüller spaces, which is used in the next section.

Let $\nu \in \operatorname{Bel}(\Gamma)$ fixed and $\tilde{R}_{\nu}(\mu)$ be the Beltrami coefficient of $f^{\mu} \circ\left(f^{\nu}\right)^{-1}$, or, in more explicit terms, by

$$
\tilde{R}_{\nu}(\mu)=\left(\frac{\mu-\nu}{1-\mu \bar{\nu}}\left(\frac{\partial f^{\nu}}{\left|\partial f^{\nu}\right|}\right)^{2}\right) \circ\left(f^{\nu}\right)^{-1}
$$


for $\mu \in \operatorname{Bel}(\Gamma)$. Then $\tilde{R}_{\nu}$ is a biholomorphic isometry of $\left(\operatorname{Bel}(\Gamma),\|\cdot\|_{\infty}\right)$ onto $\left(\operatorname{Bel}\left(\Gamma^{\nu}\right),\|\cdot\|_{\infty}\right)$ and maps $\nu$ to the origin of $\operatorname{Bel}\left(\Gamma^{\nu}\right)$. Let us call the map $\tilde{R}_{\nu}$ the right translation map for $\nu$. Let $R_{[\nu]}$ be the projection of $R_{\nu}$ by the canonical projection $\varpi: \operatorname{Bel}(\Gamma) \rightarrow T(\Gamma)$, that is,

$$
R_{[\nu]}([\mu])=\left[R_{\nu}(\mu)\right]
$$

for $[\mu] \in T(\Gamma)$. By the definition of the Teichmüller equivalence relation (1.1), $R_{[\nu]}$ is well-defined. It follows that for each $\tau \in T(\Gamma), R_{\tau}$ is a biholomorphic map of $T(\Gamma)$ onto $T\left(\Gamma^{\tau}\right)$. We also call the map $R_{\tau}$ the right translation map for $\tau$.

Let us show the homeomorphy of right translation maps in $T^{p}(\Gamma)$. Note that we do not need to assume that $\Gamma$ satisfies Lehner's condition.

Proposition 5.1. For each $\tau \in T^{p}(\Gamma)$, the right translation map $R_{\tau}$ is a homeomorphism of $\left(T^{p}(\Gamma), \ell_{p, \infty}(\Gamma)\right)$ onto $\left(T^{p}\left(\Gamma^{\tau}\right), \ell_{p, \infty}\left(\Gamma^{\tau}\right)\right)$.

Proof. Given $\tau \in T^{p}(\Gamma)$, let $\nu=\sigma(\tau)$. For every $\tau_{1}, \tau_{2} \in T^{p}(\Gamma)$, take $\mu_{k} \in$ $\varpi^{-1}\left(\tau_{k}\right) \cap \operatorname{Ael}^{p}(\Gamma)$ arbitrarily for $k=1,2$. From Theorem 2.4, $\nu$ belongs to $\operatorname{Ael}^{p}(\Gamma)$. By a simple computation, we have

$$
\left|\frac{R_{\nu}\left(\mu_{1}\right)-R_{\nu}\left(\mu_{2}\right)}{1-R_{\nu}\left(\mu_{1}\right) \overline{R_{\nu}\left(\mu_{2}\right)}}\right| \circ f^{\nu}=\left|\frac{\mu_{1}-\mu_{2}}{1-\mu_{1} \bar{\mu}_{2}}\right| .
$$

If we set $\tau_{2}=\tau$ and $\mu_{2}=\nu$, then we have $R_{\tau}\left(\tau_{1}\right)$ belongs to $T^{p}(\Gamma)$, that is, $R_{\tau}$ maps $T^{p}(\Gamma)$ into $T^{p}\left(\Gamma^{\tau}\right)$. It follows from formula $(2.3)$ that

$$
\begin{aligned}
\left\|\frac{R_{\nu}\left(\mu_{1}\right)-R_{\nu}\left(\mu_{2}\right)}{1-R_{\nu}\left(\mu_{1}\right) \overline{R_{\nu}\left(\mu_{2}\right)}}\right\|_{p}^{p} & =\iint_{N}\left|\frac{\mu_{1}(w)-\mu_{2}(w)}{1-\mu_{1}(w) \overline{\mu_{2}(w)}}\right|^{p} \rho\left(f^{\nu}(w)\right)^{2} J_{f^{\nu}}(w) d u d v \\
& \leq C\left\|\frac{\mu_{1}-\mu_{2}}{1-\mu_{1} \overline{\mu_{2}}}\right\|_{p}^{p} .
\end{aligned}
$$

Since $R_{\nu}$ is an isometry in $\|\cdot\|_{\infty}$, we obtain

$$
\ell_{p, \infty}\left(\Gamma^{\tau}\right)\left(R_{\tau}\left(\tau_{1}\right), R_{\tau}\left(\tau_{2}\right)\right) \leq\left\|\frac{R_{\nu}\left(\mu_{1}\right)-R_{\nu}\left(\mu_{2}\right)}{1-R_{\nu}\left(\mu_{1}\right) \overline{R_{\nu}\left(\mu_{2}\right)}}\right\|_{p, \infty} \leq \max \left\{C^{\frac{1}{p}}, 1\right\}\left\|\frac{\mu_{1}-\mu_{2}}{1-\mu_{1} \overline{\mu_{2}}}\right\|_{p, \infty} .
$$

Recall that $\mu_{k}$ is taken arbitrarily in $\varpi^{-1}\left(\tau_{k}\right) \cap \operatorname{Ael}^{p}(\Gamma)$ for $k=1,2$ and $C$ depends only on $\|\nu\|_{\infty}$. Hence $R_{\tau}$ is a continuous map of $T^{p}(\Gamma)$ into $T^{p}\left(\Gamma^{\tau}\right)$. Noting that $R_{\tau}^{-1}=$ $R_{\left[\nu^{-1}\right]}$, it follows immediately that $R_{\tau}$ is bijective and that $R_{\tau}^{-1}$ is also continuous.

In order to prove the biholomorphy of right translation maps, we use the following lemma that indicates two equivalent conditions for the holomorphy of maps between Banach spaces.

Lemma 5.2. Let $E, F$ be two Banach spaces and $U \subset E$ be a open set. Then a map $f: U \rightarrow F$ is holomorphic if and only if it satisfies one of the following two conditions:

(1) The map $f: U \rightarrow F$ is continuous and for every $x \in U$ and $e \in E$, the map $w \mapsto f(x+w e)$ is a holomorphic map on an open neighborhood of the origin in $\mathbf{C}$ with values in $F$;

(2) The map $f: U \rightarrow F$ is continuous and there exists a total subset $A$ of the dual $F^{*}$ such that, for every $\alpha \in A$, the function $\alpha \circ f: U \rightarrow \mathbf{C}$ is holomorphic.

Here a subset $A \subset F^{*}$ is called total if $\alpha(x)=0$ for every $\alpha \in A$ implies $x=0$. 
It is used in the proof of the biholomorphy of right translation maps on $T(\Gamma)$ (cf. Chapter V.5 in [16]). For the detail of this lemma, refer to Chapter 3.3 in [3].

Proposition 5.3. Let $p \geq 2$ and $\Gamma$ be a Fuchsian group satisfying Lehner's condition. Then for each $\tau \in T^{p}(\Gamma)$, the right translation map $R_{\tau}$ is a biholomorphic map of $T^{p}(\Gamma)$ onto $T^{p}\left(\Gamma^{\tau}\right)$. Moreover, $T^{p}\left(\Gamma^{\tau}\right)$ is biholomorphically equivalent to $T^{p}(\Gamma)$.

Proof. Fix $\tau, \eta \in T^{p}(\Gamma)$ and $\varphi \in A^{p}\left(\Gamma^{\tau}\right)$. Let $\beta_{\tau}$ be the Bers embedding of $T^{p}\left(\Gamma^{\tau}\right)$ into $A^{p}\left(\Gamma^{\tau}\right)$ and $H=\beta_{\tau} \circ R_{\tau} \circ \beta^{-1}$. Since $H^{-1}=\beta \circ R_{\tau^{-1}} \circ \beta_{\tau}^{-1}$, it is sufficient to show that $H$ is holomorphic from $\left(\beta\left(T^{p}(\Gamma)\right),\|\cdot\|_{p}\right)$ into $\left(A^{p}\left(\Gamma^{\tau}\right),\|\cdot\|_{p}\right)$.

For $z \in \Delta^{*}$ and $\psi \in A^{p}\left(\Gamma^{\tau}\right)$, define $\alpha_{z}(\psi)=\psi(z)$. By Proposition 4.2,

$$
\sup _{\psi \in A^{p}\left(\Gamma^{\tau}\right)} \frac{\left|\alpha_{z}(\psi)\right|}{\|\psi\|_{p}} \leq \rho(z)^{2} \sup _{\psi \in A^{p}\left(\Gamma^{\tau}\right)} \frac{\|\psi\|_{\infty}}{\|\psi\|_{p}} \leq C_{p} \rho(z)^{2}<\infty .
$$

Hence $\alpha_{z}$ is a bounded linear functional on $A^{p}\left(\Gamma^{\tau}\right)$ and the set $A=\left\{\alpha_{z} \mid z \in \Delta^{*}\right\}$ is a total subset in the dual space of $A^{p}\left(\Gamma^{\tau}\right)$. Since $\beta\left(T^{p}(\Gamma)\right)$ is open in $A^{p}(\Gamma)$, there exists a positive number $r>0$ such that $\beta(\eta)+w \varphi$ lies in $\beta\left(T^{p}(\Gamma)\right)$ for every $w \in\{|\zeta|<r\}$. We apply condition (2) of Lemma 5.2 to the function

$$
w \mapsto \hat{H}(w)=H(\beta(\eta)+w \varphi) .
$$

The set $U$ in condition (2) is now the neighborhood $\{|\zeta|<r\}$. Furthermore, let $F=A^{p}(\Gamma)$ and $\alpha=\alpha_{z}$. Since $R_{\tau}$ is holomorphic on $T(\Gamma)$, it follows from Lemma 5.2 that the function

$$
w \mapsto \alpha_{z} \circ \hat{H}(w)=H(\beta(\eta)+w \varphi)(z)
$$

is holomorphic in $U$ for every $z \in \Delta^{*}$. By Theorem 4.4 and Proposition 5.1, the function $\hat{H}$ is continuous from $\left(U, d_{E}\right)$ into $\left(A^{p}\left(\Gamma^{\tau}\right),\|\cdot\|_{p}\right)$, where $d_{E}$ is the Euclidean metric on C. Hence condition (2) of Lemma 5.2, the function $\hat{H}$ is holomorphic from $\left(U, d_{E}\right)$ into $\left(A^{p}\left(\Gamma^{\tau}\right),\|\cdot\|_{p}\right)$. Using this fact, we conclude from condition (1) of Lemma 5.2 that $H$ is holomorphic in $\beta\left(T^{p}(\Gamma)\right)$.

\section{Coincidence of two canonical distances on $T^{p}(\Gamma)$}

In this section, we show the coincidence of two canonical distances on the $p$ integrable Teichmüller space of a Fuchsian group satisfying Lehner's condition, which is an application of Theorem 4.4.

For the proof, let us introduce another representation of the Teichmüller space. For a Fuchsian group $\Gamma$ acting on $\Delta$, let $\mathcal{R}=\Delta / \Gamma$ and $\pi: \Delta \longrightarrow \mathcal{R}$ be the canonical projection. It is known that a function on $\Delta$ is a $(-1,1)$-differential for $\Gamma$ if and only if its projection to $\mathcal{R}$ is a $(-1,1)$-differential on $\mathcal{R}$ (see [16], Chapter IV.3.6). We identify $\operatorname{Bel}(\Gamma)$ with the set of measurable $(-1,1)$-differentials on $\mathcal{R}$. Denote $\mathrm{QC}(\Gamma)$ as the set of quasiconformal mappings on $\mathcal{R}$. There exists a one-to-one correspondence

$$
\operatorname{Bel}(\Gamma) \ni \mu \longleftrightarrow \hat{f}^{\mu} \in \mathrm{QC}(\Gamma)
$$

where $\hat{f}^{\mu}$ is the quasiconformal mapping on $\mathcal{R}$ with Beltrami coefficient $\mu$. Then $T(\Gamma)$ can be regarded as the quotient space of $\mathrm{QC}(\Gamma)$ by the homotopy relative to the boundary, that is, two quasiconformal mappings $f, g \in \mathrm{QC}(\Gamma)$ is equivalent if there exists a conformal map $h: f(\mathcal{R}) \rightarrow g(\mathcal{R})$ such that

$$
h \circ f \sim g
$$


with fixing their boundary values. A point of $T(\Gamma)$ represented by $f \in \mathrm{QC}(\Gamma)$ is denoted by $[f]$.

For $f \in \mathrm{QC}(\Gamma)$, let

$$
K(f)=\frac{1+\|\mu\|_{\infty}}{1-\|\mu\|_{\infty}}
$$

Then $K(f)$ is said to be the maximal dilatation of $f$. The Teichmüller distance between the points $p$ and $q$ of $T(\Gamma)$ is defined as

$$
d_{T(\Gamma)}(p, q)=\frac{1}{2} \inf \log K\left(g \circ f^{-1}\right),
$$

where the infimum is taken over all $f \in p$ and $g \in q$. Then $\left(T(\Gamma), d_{T(\Gamma)}\right)$ is a complete and contractible metric space (cf. [16], Section V.3). The Teichmüller distance on $T^{p}(\Gamma)$ means that the restriction of $d_{T(\Gamma)}$ to $T^{p}(\Gamma)$.

Let $M$ be a complex manifold and let $H(\Delta, M)$ be the set of holomorphic maps from $\Delta$ into $M$. For $p, q \in M$, let

$$
d_{1}(p, q)=\frac{1}{2} \log \frac{1+r}{1-r}
$$

where $r$ denotes the infimum of $s \geq 0$ such that there exists $f \in H(\Delta, M)$ satisfying $f(0)=p$ and $f(s)=q$. If no such $f$ exists in $H(\Delta, M)$, then we define $d_{1}(p, q)=\infty$. Let

$$
d_{n}(p, q)=\inf \sum_{i=1}^{n} d_{1}\left(p_{i-1}, p_{i}\right),
$$

where the infimum is taken over all chains of points $p_{0}=p, p_{1}, \ldots, p_{n}=q$ in $M$. Clearly, $d_{n+1} \leq d_{n}$ for all $n>0$. The Kobayashi pseudo-distance on $M$ is defined as

$$
d_{K}(p, q)=\lim _{n \rightarrow \infty} d_{n}(p, q) .
$$

If $d_{K}$ is non-degenerate, i.e. if $d_{K}(p, q)=0$ implies $p=q$, then $d_{K}$ is called the Kobayashi distance on $M$.

The Kobayashi pseudo-distance has an important property in terms of the contraction of the distance.

Proposition 6.1. Let $M$ and $M^{\prime}$ be two complex manifolds and $d_{K, M}$ and $d_{K, M^{\prime}}$ denote the Kobayashi pseudo-distances on $M$ and $M^{\prime}$, respectively. Then for every holomorphic map $F$ from $M$ into $M^{\prime}$ and every two points $p, q \in M$,

$$
d_{K, M^{\prime}}(F(p), F(q)) \leq d_{K, M}(p, q) .
$$

If $F$ is a biholomorphic map between $M$ and $M^{\prime}$, then $F$ is an isometry in the Kobayashi pseudo-distance. Furthermore, if both $M$ and $M^{\prime}$ are $\Delta$, then Proposition 6.1 is nothing but the Schwarz-Pick lemma.

We write the fact about these two canonical distances on the Teichmüller space, which we mentioned in Section 1:

Theorem 6.2 ([10]). For every Fuchsian group $\Gamma$, the Teichmüller distance on $T(\Gamma)$ coincides with the Kobayashi distance.

Next, we need to modify the preparation in [21], Section 3. For every $\tau \in T(\Gamma)$, there always exists a quasiconformal mapping of $\tau$ that has the smallest maximal dilatation in $\tau$, which is called extremal in $\tau$. If $\tau$ has the property as in Theorem 6.4 
below, then the extremal mapping is uniquely determined and can be represented concretely.

Definition 6.3. For $\tau \in T(\Gamma)$, let $f_{0}$ be an extremal quasiconformal mapping of $\tau$. An element $f_{1}$ of $\tau$ is called a frame mapping for $\tau$ if $f_{1}$ satisfies the following condition: There exists a compact subset $E \subset \mathcal{R}$ such that

$$
K\left(\left.f_{1}\right|_{\mathcal{R} \backslash E}\right)<K\left(f_{0}\right) .
$$

If there exists a frame mapping in $\tau$, then $\tau$ is called a Strebel point.

The set of Strebel points is open and dense in $T(\Gamma)$ (see p.106 in [12]).

Theorem 6.4. (Strebel's Frame Mapping Theorem, Teichmüller's Uniqueness Theorem) If a point $\tau \in T(\Gamma)$ is a Strebel point, then it has the unique extremal mapping $f_{0}$ with Beltrami coefficient of the form

$$
k \frac{\bar{\varphi}}{|\varphi|}
$$

where $0<k<1$ and $\varphi$ is a holomorphic quadratic differential on $\mathcal{R}$ with

$$
\iint_{\mathcal{R}}|\varphi(z)| d x d y=1
$$

The proof can be found in [12]. A quasiconformal mapping with Beltrami coefficient of form (6.2) is said to be a Teichmüller mapping.

The next theorem states that the maximal dilatation of each Teichmüller mapping can be estimated by every representative in its Teichmüller equivalence class.

Proposition 6.5. (Fundamental Inequality) Let $f_{0}$ be a Teichmüller mapping with Beltrami coefficient $k_{0} \overline{\varphi_{0}} /\left|\varphi_{0}\right|$, where $0<k_{0}<1$ and $\varphi_{0}$ is a holomorphic quadratic differential on $\mathcal{R}$ with $\iint_{\mathcal{R}}\left|\varphi_{0}(z)\right| d x d y=1$. Then for every $\hat{f}^{\nu} \in\left[f_{0}\right]$,

$$
K\left(f_{0}\right) \leq \iint_{\mathcal{R}} \frac{\left|1+\nu(z) \frac{\varphi_{0}(z)}{\left|\varphi_{0}(z)\right|}\right|^{2}}{1-|\nu(z)|^{2}}\left|\varphi_{0}(z)\right| d x d y .
$$

The proof can be found in [11] and [12].

An example of frame mappings is the asymptotically conformal map. A quasiconformal mapping $f$ on a Riemann surface $\mathcal{R}$ is called asymptotically conformal if for every $\varepsilon>0$, there exists a compact subset $E$ of $\mathcal{R}$ such that

$$
K\left(\left.f\right|_{\mathcal{R} \backslash E}\right)<1+\varepsilon .
$$

It follows immediately that for $\mu \in \operatorname{Bel}(\Gamma), \hat{f}^{\mu}$ is asymptotically conformal if and only if for every $\varepsilon>0$, there exists a compact subset $E$ of $\mathcal{R}$ such that

$$
\left\|\left.\mu\right|_{\pi^{-1}(\mathcal{R} \backslash E)}\right\|_{\infty}<\varepsilon \text {. }
$$

If $\mu$ satisfies this condition, it is said that $\mu$ vanishes at infinity. Let $\operatorname{Bel}_{0}(\Gamma)$ be the set of Beltrami coefficints vanishing at infinity.

We call a Teichmüller equivalence class $\tau$ of $T(\Gamma)$ asymptotically conformal if $\tau$ has an asymptotically conformal map of $\Delta$ onto itself. Let $T_{0}(\Gamma)$ be the set of asymptotically conformal classes of $T(\Gamma)$. For $\varphi \in \mathcal{B}(\Gamma)$, it is said that $\varphi$ vanishes at infinity if for every $\varepsilon>0$, there exists a compact subset $E \subset \mathcal{R}$ such that

$$
\left\|\left.\varphi\right|_{\pi^{-1}(\mathcal{R} \backslash E)}\right\|_{\infty}<\varepsilon .
$$


Let $\mathcal{B}_{0}(\Gamma)$ be the set of bounded holomorphic quadratic differentials vanishing at infinity. Since $\mathcal{B}_{0}(\Gamma)$ is a closed subspace of $\mathcal{B}(\Gamma), \mathcal{B}_{0}(\Gamma)$ is a Banach space. In [7], it is shown that $\beta\left(T_{0}(\Gamma)\right)=\beta(T(\Gamma)) \cap \mathcal{B}_{0}(\Gamma)$. Thus $T_{0}(\Gamma)$ has a complex structure modeled on $\mathcal{B}_{0}(\Gamma)$, that is, $T_{0}(\Gamma)$ is a closed submanifold of $T(\Gamma)$. It is known that the Teichmüller distance on $T_{0}(\Gamma)$ coincides with the Kobayashi distance (cf. [8]).

It follows that there exists the following inclusion relation:

Proposition 6.6. Let $p \geq 1$. If a Fuchsian group $\Gamma$ satisfies Lehner's condition, then

$$
A^{p}(\Gamma) \subset \mathcal{B}_{0}(\Gamma)
$$

Proof. Let $L^{p}(\Gamma)$ be the Banach space of measurable quadratic differentials on $\Delta^{*}$ with finite hyperbolic $L^{p}$-norm $\|\cdot\|_{p}$ and $L_{c}(\Gamma)$ be the set of measurable quadratic differentials with compact support. It clearly follows that $L_{c}(\Gamma)$ is dense in $L^{p}(\Gamma)$. The Bergmann projection

$$
P \varphi(z)=\frac{12}{\pi} \iint_{\Delta^{*}} \rho_{*}(\zeta)^{-2} \frac{\varphi(\zeta)}{(z \bar{\zeta}-1)^{4}} d \xi d \eta \quad\left(z \in \Delta^{*}\right)
$$

is a bounded liner operator of $L^{p}(\Gamma)$ onto $A^{p}(\Gamma)$ (see [14], Theorem III.3.2). Hence $P\left(L_{c}(\Gamma)\right)$ is dense in $A^{p}(\Gamma)$. Similarly, $P\left(L_{c}(\Gamma)\right)$ is also dense in $\mathcal{B}_{0}(\Gamma)$. Noting that $\mathcal{B}_{0}(\Gamma)$ is closed in $\mathcal{B}(\Gamma)$, it follows from these two densenesses and Proposition 4.2 that $A^{p}(\Gamma) \subset \mathcal{B}_{0}(\Gamma)$.

This proposition implies that $T^{p}(\Gamma) \subset T_{0}(\Gamma)$.

Let $A(\mathcal{R})$ be the Banach space of integrable holomorphic quadratic differentials on a Riemann surface $\mathcal{R}$ and $A_{1}(\mathcal{R})$ be the unit ball of $A(\mathcal{R})$. If a sequence $\left\{E_{n}\right\}$ of relatively compact domains of $\mathcal{R}$ satisfies the following four conditions, then the sequence is called an exhaustion of $\mathcal{R}$ :

(1) $\bar{E}_{n} \subset E_{n+1}$ for all $n \geq 1$;

(2) $\bigcup_{n=1}^{\infty} E_{n}=\mathcal{R}$;

(3) The boundary of $E_{n}$ is the disjoint union of analytic Jordan curves of $\mathcal{R}$;

(4) Each connected component of $\mathcal{R} \backslash E_{n}$ is non-compact.

It is well-known that every non-compact Riemann surface $\mathcal{R}$ has an exhaustion (cf. $[?, ?])$.

Lemma 6.7. Let $\left\{E_{n}\right\}$ be an exhaustion of $\mathcal{R}$ and $\varphi_{n}$ be a holomorphic quadratic differential of $A_{1}\left(E_{n}\right)$ for each $n$. Then there exists a subsequence of $\left\{\varphi_{n}\right\}$ that converges locally uniformly to a holomorphic quadratic differential $\varphi \in A_{1}(\mathcal{R})$ in $\mathcal{R}$.

This lemma clearly holds by changing $A_{1}(\Delta)$ to $A_{1}(\mathcal{R})$ in the proof of Lemma 3.5 in $[21]$.

Now let us show the main theorem in this section.

Theorem 6.8. Let $p \geq 2$ and $\Gamma$ be a Fuchsian group satisfying Lehner's condition. Then the Teichmüller distance on $T^{p}(\Gamma)$ coincides with the Kobayashi distance.

Proof. Let $d_{T^{p}(\Gamma)}=\left.d_{T(\Gamma)}\right|_{T^{p}(\Gamma)}$ and $d_{K^{p}(\Gamma)}$ be the Kobayashi pseudo-distance on $T^{p}(\Gamma)$. The inclusion map $\iota: T^{p}(\Gamma) \longrightarrow T(\Gamma)$ is holomorphic. Indeed, by Proposition 4.2, the identity map $i d_{\beta\left(T^{p}(\Gamma)\right)}=\beta^{-1} \circ \iota \circ \beta$ is a continuous liner map of $\left(\beta\left(T^{p}(\Gamma)\right),\|\cdot\|_{p}\right)$ into $\left(\mathcal{B}(\Gamma),\|\cdot\|_{\infty}\right)$. Then the holomorphy of $\iota$ clearly holds. Hence 
it follows from Proposition 6.1 and Theorem 6.2 that

$$
d_{T^{p}(\Gamma)}\left(\tau_{1}, \tau_{2}\right)=d_{T(\Gamma)}\left(\tau_{1}, \tau_{2}\right)=d_{K(\Gamma)}\left(\iota\left(\tau_{1}\right), \iota\left(\tau_{2}\right)\right) \leq d_{K^{p}(\Gamma)}\left(\tau_{1}, \tau_{2}\right)
$$

for every $\tau_{1}, \tau_{2} \in T^{p}(\Gamma)$, where $d_{K(\Gamma)}$ is the Kobayashi distance on $T(\Gamma)$. Hence we have $d_{T^{p}(\Gamma)} \leq d_{K^{p}(\Gamma)}$.

Let us show that the opposite inequality holds. From Proposition 5.3, $R_{\tau}$ is a biholomorphic map from $T^{p}(\Gamma)$ onto $T^{p}\left(\Gamma^{\tau}\right)$ for every $\tau \in T^{p}(\Gamma)$. Hence by Proposition 6.1, it is sufficient to show that for each non-base point $\tau \in T^{p}(\Gamma)$,

$$
d_{K^{p}(\Gamma)}(0, \tau) \leq d_{T^{p}(\Gamma)}(0, \tau) .
$$

Recall that $\sigma(\tau)$ is the Beltrami coefficient of the Douady-Earle extension $E(\tau)$ for $\tau$. By Theorem 2.4, $\sigma(\tau)$ belongs to $\operatorname{Ael}^{p}(\Gamma)$. Since $T^{p}(\Gamma)$ is contained in $T_{0}(\Gamma)$, it follows from Theorem 4 in [9] that $\sigma(\tau)$ also belongs to $\operatorname{Bel}_{0}(\Gamma)$. Let $f$ and $\mu$ be the projection of $E(\tau)$ and $\sigma(\tau)$ by $\pi$, respectively. By Strebel's frame mapping theorem, $\tau$ has a unique extremal mapping $f_{0}$ on $R$. Note that $1<K_{0}=K\left(f_{0}\right)<K(f)$.

Let $\left\{E_{n}\right\}$ denote an exhaustion of $\mathcal{R}$. Since $f$ is a frame mapping, there exists a number $N \in \mathbf{N}$ such that

$$
K\left(\left.f\right|_{\mathcal{R} \backslash \bar{E}_{n}}\right)<K_{0}
$$

for each $n \geq N$. For such $n$, we consider the Teichmüller space of $E_{n}$, denoted by $T\left(E_{n}\right)$. Similarly to $T(\Gamma)$, it is defined as the quotient space of the set of quasiconformal mappings on $E_{n}$ by the homotopy relative to $\partial E_{n}$. Let $\tilde{f}_{n}$ be an extremal mapping in $\left[\left.f\right|_{E_{n}}\right] \in T\left(E_{n}\right)$. Then $K\left(\tilde{f}_{n}\right) \geq K_{0}$. Indeed, suppose to the contrary. Set

$$
f_{n}(z)= \begin{cases}\tilde{f}_{n}(z), & z \in \bar{E}_{n}, \\ f(z), & z \in \mathcal{R} \backslash \bar{E}_{n} .\end{cases}
$$

Since $\tilde{f}_{n}$ belongs to $\left[\left.f\right|_{E_{n}}\right], \tilde{f}_{n}$ agrees with $f$ on $\partial E_{n}$. Hence $f_{n}$ is a homeomorphism of $\mathcal{R}$. Moreover, $f$ and $\tilde{f}_{n}$ are quasiconformal and $\partial E_{n}$ has zero measure. Hence $f_{n}$ is a quasiconformal mapping on $\mathcal{R}$ and $f_{n} \in \tau$. Thus we have $K_{0} \leq K\left(f_{n}\right)$. However, from inequality (6.3), we have

$$
K\left(f_{n}\right)=\max \left\{K\left(\left.f\right|_{\mathcal{R} \backslash \bar{E}_{n}}\right), K\left(\tilde{f}_{n}\right)\right\}<K_{0} .
$$

This contradicts the assumption. From this result, we have

$$
K\left(\left.f\right|_{E_{n} \backslash \bar{E}_{N}}\right) \leq K\left(\left.f\right|_{\mathcal{R} \backslash \bar{E}_{N}}\right)<K_{0} \leq K\left(\tilde{f}_{n}\right) .
$$

Thus $\left.f\right|_{E_{n}}$ is a frame mapping for $\left[\left.f\right|_{E_{n}}\right] \in T\left(E_{n}\right)$. By Strebel's frame mapping theorem, $\tilde{f}_{n}$ is the Teichmüller mapping with Beltrami coefficient $k_{n} \overline{\varphi_{n}} /\left|\varphi_{n}\right|$ where $0<k_{n}<1$ and $\varphi_{n}$ is a quadratic differential on $E_{n}$ with $\iint_{E_{n}}\left|\varphi_{n}\right|=1$. Let $\mu_{n}$ be the Beltrami coefficient of $f_{n}$ and $K_{n}=K\left(f_{n}\right)$. Then it is easily seen that

(a) $K_{n}>K_{0}$,

(b) $\left[\mu_{n}\right]=[\mu]$,

(c) for every $t \in \Delta,\left[t \mu_{n}\right] \in T^{p}(\Gamma)$

for each $n$.

Let $g(t)=\left[t \mu_{n} /\left\|\mu_{n}\right\|_{\infty}\right]$ for $t \in \Delta$. It follows from condition (c) that $g$ maps $\Delta$ into $T^{p}(\Gamma)$. Furthermore, $g$ is holomorphic on $\Delta$ and $g(0)=0, g\left(\left\|\mu_{n}\right\|_{\infty}\right)=\left[\mu_{n}\right]$. By 
formula (b) and the definition of the Kobayashi pseudo-distance,

$$
d_{K^{p}(\Gamma)}(0, \tau) \leq d_{1}(0, \tau)=d_{1}\left(0,\left[\mu_{n}\right]\right) \leq \frac{1}{2} \log \frac{1+\left\|\mu_{n}\right\|_{\infty}}{1-\left\|\mu_{n}\right\|_{\infty}}=\frac{1}{2} \log K_{n} .
$$

Let us show that there exists a subsequence of $\left\{K_{n}\right\}$ tending to $K_{0}$. From Proposition 6.7, there exists a subsequence of $\left\{\varphi_{n}\right\}$ which converges locally uniformly in $\mathcal{R}$ to a holomorphic quadratic differential $\varphi^{*} \in A_{1}(\mathcal{R})$. We write this subsequence as $\left\{\varphi_{n}\right\}$ again.

To show $\left\|\varphi^{*}\right\|_{1}>0$, suppose to the contrary that $\left\|\varphi^{*}\right\|_{1}=0$. Then $\left\{\varphi_{n}\right\}$ converges locally uniformly to 0 in $\mathcal{R}$. Take $\varepsilon>0$ arbitrarily. Since $\lim _{n \rightarrow \infty} \iint_{\bar{E}_{N}}\left|\varphi_{n}\right|=0$, there exists a number $N^{\prime} \in \mathbf{N}$ such that

$$
\iint_{\bar{E}_{N}}\left|\varphi_{n}\right|<\varepsilon
$$

for $n>N^{\prime}$. Let $\tilde{N}=\max \left\{N, N^{\prime}\right\}$. For any $n>\tilde{N}$, the fundamental inequality implies that

$$
K\left(\tilde{f}_{n}\right) \leq \iint_{E_{n}} \frac{\left|1+\mu \frac{\varphi_{n}}{\left|\varphi_{n}\right|}\right|^{2}}{1-|\mu|^{2}}\left|\varphi_{n}\right| d x d y .
$$

We estimate the right-hand integral by dividing $E_{n}$ into $\bar{E}_{N}$ and $E_{n} \backslash \bar{E}_{N}$.

$$
\begin{aligned}
\iint_{\bar{E}_{N}} \frac{\left|1+\mu \frac{\varphi_{n}}{\left|\varphi_{n}\right|}\right|^{2}}{1-|\mu|^{2}}\left|\varphi_{n}\right| d x d y & \leq \iint_{\bar{E}_{N}} \frac{1+|\mu|}{1-|\mu|}\left|\varphi_{n}\right| d x d y \\
& \leq \frac{1+\|\mu\|_{\infty}}{1-\|\mu\|_{\infty}} \iint_{\bar{E}_{N}}\left|\varphi_{n}\right| d x d y<K \varepsilon ; \\
\iint_{E_{n} \backslash \bar{E}_{N}} \frac{\left|1+\mu \frac{\varphi_{n}}{\left|\varphi_{n}\right|}\right|^{2}}{1-|\mu|^{2}}\left|\varphi_{n}\right| d x d y & \leq \frac{1+\left\|\left.\mu\right|_{\mathcal{R} \backslash \bar{E}_{N}}\right\|_{\infty}}{1-\left\|\left.\mu\right|_{\mathcal{R} \backslash \bar{E}_{N}}\right\| \|_{\infty}} \iint_{E_{n} \backslash \bar{E}_{N}}\left|\varphi_{n}\right| d x d y \\
& \leq K\left(\left.f\right|_{\mathcal{R} \backslash \bar{E}_{N}}\right) .
\end{aligned}
$$

Recall that $K=K(f)=\left(1+\|\mu\|_{\infty}\right) /\left(1-\|\mu\|_{\infty}\right)$. It follows from these inequalities that $K\left(\tilde{f}_{n}\right)<K \varepsilon+K\left(\left.f\right|_{\mathcal{R} \backslash \bar{E}_{N}}\right)$. Taking $\varepsilon \rightarrow 0$, we obtain

$$
\limsup _{n \rightarrow \infty} K\left(\tilde{f}_{n}\right) \leq K\left(\left.f\right|_{\mathcal{R} \backslash \bar{E}_{N}}\right) .
$$

By inequality (6.3), we have

$$
\limsup _{n \rightarrow \infty} K_{n} \leq \max \left\{\limsup _{n \rightarrow \infty} K\left(\tilde{f}_{n}\right), K\left(\left.f\right|_{\mathcal{R} \backslash \bar{E}_{N}}\right)\right\}<K_{0}
$$

However, this contradicts inequality (a). Therefore, $\left\|\varphi^{*}\right\|_{1}>0$.

Since $0<k_{n}<1$ for all $n,\left\{k_{n}\right\}$ has a convergent subsequence. Let $k^{*}$ be the limit of this subsequence and $\mu^{*}=k^{*} \overline{\varphi^{*}} /\left|\varphi^{*}\right|$. Since $\left\|\varphi^{*}\right\|_{1}>0$, $\mu^{*}$ is welldefined and $\left\{\mu_{n}\right\}$ converges pointwise to $\mu^{*}$ on $\mathcal{R}$. Because $K\left(f_{n}\right)=K_{n} \leq K$ for every $n$, we have $\left\|\mu_{n}\right\|_{\infty} \leq\|\mu\|_{\infty}<1$. It follows from Lemma V.3.1 in [16] that $\left[\mu^{*}\right]=[\mu]$. From the uniqueness of extremal mappings, we have $k^{*}=k_{0}$. Noting that $K_{0}=\left(1+k_{0}\right) /\left(1-k_{0}\right)$, this implies that $\left\{K_{n}\right\}$ has a subsequence converging to $K_{0}$. Therefore, $d_{T^{p}(\Gamma)}=d_{K^{p}(\Gamma)}$.

The essential point of this proof is that the projection of the Douady-Earle extension for each point of $T^{p}(\Gamma)$ is a frame mapping, which follows from that $T^{p}(\Gamma)$ 
is contained in $T_{0}(\Gamma)$. Recall that for every Fuchsian group $\Gamma, T_{0}(\Gamma)$ is a closed submanifold of $T(\Gamma)$. It is known that the right translation map $R_{\tau}$ for each $\tau \in T_{0}(\Gamma)$ is biholomorphic of $T_{0}(\Gamma)$ onto $T_{0}\left(\Gamma^{\tau}\right)$. Therefore, the following result holds similarly to the above theorem:

Theorem 6.9. For every Fuchsian group $\Gamma$, the Teichmüller distance on $T_{0}(\Gamma)$ coincides with the Kobayashi distance.

As we mentioned previously, this result is shown in [8]. Hence we obtained another proof.

Remark. Recall that the Teichmüller distance $d_{T^{p}(\Gamma)}$ on $T^{p}(\Gamma)$ is the restriction of the Teichmüller distance on $T(\Gamma)$ to $T^{p}(\Gamma)$. On the other hand, the Kobayashi distance $d_{K^{p}(\Gamma)}$ on $T^{p}(\Gamma)$ is determined by $d_{1^{-}}$lengths of paths on $T^{p}(\Gamma)$, that is, $\left(T^{p}(\Gamma), d_{K^{p}(\Gamma)}\right)$ is a path metric space. These facts imply that the topology induced by $d_{T^{p}(\Gamma)}$ is weaker than or equal to the one induced by $d_{K^{p}(\Gamma)}$. Theorem 6.8 means that these topologies coincide and that $\left(T^{p}(\Gamma), d_{T^{p}(\Gamma)}\right)$ is a path metric space.

Acknowledgment. The author would like to give heartful thanks to Professor Katsuhiko Matsuzaki whose enormous support and insightful comments were invaluable during the course of my study. The author is also indebt to the referees for their careful reading and valuable comments.

\section{References}

[1] Ahlfors, L. V.: Quasiconformal reflections. - Acta Math. 109, 1963, 291-301.

[2] Astala, K., and M. Zinsmeister: Teichmüller spaces and BMOA. - Math. Ann. 289, 1991, 613-625.

[3] Bourbaki, N.: Variétiés différentielles et analytiques. - Eléments de mathématique XXXIII, Fascicule de résultats-Paragraghes 1 á 7, Hermann, 1967.

[4] Choquet, G.: Sur un type de transformation analytique généralisant la réprésentation conforme et définie au moyen de foctions harmoniques. - Bull. Sci. Math. 69, 1945, 156.

[5] CUI, G.: Integrably asymptotic affine homeomorphisms of the circle and Teichmüller spaces. Sci. China Ser. A 43, 2000, 267-279.

[6] Douady, A., and C. J. Earle: Conformally natural extension of homeomorphisms of the circle. - Acta Math. 157, 1986, 23-48.

[7] Earle, C. J., F. P. Gardiner, and N. Lakic: Asymptotic Teichmüller space. Part I: The complex structure. - Contemp. Math. 256, 2000, 17-38.

[8] Earle, C. J., F. P. Gardiner, and N. Lakic: Asymptotic Teichmüller space. Part II: The metric structure. - Contemp. Math. 355, 2004, 187-219.

[9] Earle, C. J., V. Markovic, and D. Saric: Barycentric extension and the Bers embedding for asymptotic Teichmüller space. - Contemp. Math. 311, 2002, 87-105.

[10] Gardiner, F. P.: Approximation of infinite dimensional Teichmüller spaces. - Trans. Amer. Math. Soc. 282, 1984, 367-383.

[11] Gardiner, F. P.: Teichmüller theory and quadratic differentials. - Pure and Applied Mathematics, John Wiley \& Sons, Inc., New York, 1987.

[12] Gardiner, F. P., and N. LAKIC: Quasiconformal Teichmüller theory. - Math. Surveys Monogr. 76, Amer. Math. Soc., 2000.

[13] Hu, J., Y. JiAng, and Z. WANG: Kobayashi's and Teichmüller's metrics on the Teichmüller space of symmetric circle homeomorphisms. - Acta Math. Sin. (Engl. Ser.) 27, 2011, 617-624. 
[14] Kra, I.: Automorphic forms and Kleinian groups. - Mathematics Lecture Note Series, W. A. Benjamin, Inc., 1972.

[15] Lehner, J.: On the boundedness of integrable automorphic forms. - Illinois J. Math. 18, 1974, $575-584$.

[16] Lehto, O.: Univalent functions and Teichmüller spaces. - Grad. Texts in Math. 109, SpringerVerlag, New York, 1987.

[17] NAG, S.: The complex analytic theory of Teichmüller spaces. - John Wiley \& Sons, Inc., New York, 1988.

[18] Niebur, D., and M. Sheingorn: Characterization of Fuchsian groups whose integrable forms are bounded. - Ann. of Math. 106, 1977, 239-258.

[19] Rajeswara RaO, K. V.: On the boundedness of p-integrable automorphic forms. - Proc. Amer. Math. Soc. 44, 1974, 278-282.

[20] TANG, S.: Some characterizations of the integrable Teichmüller space. - Sci. China Math. 56, $2013,541-551$.

[21] Yanagishita, M.: Teichmüller distance and Kobayashi distance on subspaces of the universal the Teichmüller distance space. - Kodai Math. J. 36, 2013, 209-227.

Received 24 February $2014 \bullet$ Revised received 14 May $2014 \bullet$ Accepted 16 May 2014 